\title{
A flexible approach for coupling NURBS patches in rotationless isogeometric analysis of Kirchhoff-Love shells
}

\author{
Laurens Coox ${ }^{\mathrm{a}, \mathrm{b}, *}$, Florian Maurin ${ }^{\mathrm{a}, \mathrm{b}}$, Francesco Greco ${ }^{\mathrm{a}, \mathrm{b}}$, Elke Deckers $^{\mathrm{a}, \mathrm{b}}$, \\ Dirk Vandepitte ${ }^{\mathrm{a}, \mathrm{b}}$, Wim Desmet $^{\mathrm{a}, \mathrm{b}}$ \\ ${ }^{a} K U$ Leuven, Department of Mechanical Engineering, Celestijnenlaan 300 box 2420, B-3001 Leuven, \\ Belgium \\ ${ }^{b}$ Member of Flanders Make
}

\begin{abstract}
This paper presents a flexible method for coupling NURBS patches in isogeometric Kirchhoff-Love shell analysis. The required $\mathrm{C}^{1}$-continuity in such a shell formulation significantly complicates the patch coupling (as compared to typical $\mathrm{C}^{0}$-cases). In the present work, the $\mathrm{C}^{0}$-part of the coupling is a global coupling in a weak sense, whereas the $\mathrm{C}^{1}$-continuity is enforced by a strong point-wise coupling in well-chosen collocation points along the interface. The coupling conditions can be derived using only mesh information, without the need for suitable penalty or stabilisation parameters. They are expressed using a master-slave formulation between the interface variables. A static condensation approach to enforce these continuity constraints results in a reduced system matrix. The proposed method can be employed for both conforming and non-conforming patch configurations, and for $\mathrm{G}^{1}$-continuous structures as well as for patches meeting at a kink. This is demonstrated for a set of problems of (dynamic) shell analysis, including both eigenvalue and boundary-value problems.
\end{abstract}

Keywords: isogeometric analysis, multipatch coupling, non-conforming patches, NURBS, Kirchhoff-Love shells

\section{Introduction}

The Finite Element Method (FEM) has been the go-to tool in industry for a multitude of Computer-Aided Engineering (CAE) purposes for several decades already. Nonetheless, the geometry descriptions employed in the FEM remain very different from those used in Computer-Aided Design (CAD). Under the hood of CAD tools are typically splinebased parametrisations, which require highly time-consuming meshing steps to translate to the element-based discretisations needed for analysis with the FEM. IsoGeometric Analysis (IGA) is a numerical modelling method introduced by Hughes et al. [1, 2] that addresses this issue by introducing CAD descriptions into a CAE framework. It can be seen as a generalisation of the FEM, replacing the traditional element-based discretisation and the associated (usually low-order polynomial) shape function expansions by CADbased mappings and associated functions. These are in the vast majority of CAD tools Non-Uniform Rational B-Splines (NURBS). IGA has already been studied extensively

*Corresponding author. Tel.: +321632 2847.

Email address: Laurens. Coox@mech.kuleuven.be (Laurens Coox) 
and applied successfully to a multitude of problem types, as illustrated by the overview presented in [3].

An application where NURBS basis functions are of particular interest is the analysis of thin shells, which can be modelled using the Kirchhoff-Love theory. For thick shells, the Reissner-Mindlin formulation is typically employed [4]. Although many shell structures in engineering practice can be regarded as thin shells, Reissner-Mindlin models are much more common in state-of-the-use finite element codes, mainly because they only require $\mathrm{C}^{0}$-continuity. Kirchhoff-Love models, on the other hand, need $\mathrm{C}^{1}$-continuous shell elements. The latter are not easily obtained when employing conventional Lagrangian shape functions, typically resulting in complex and expensive implementations [5-7]. NURBS functions, however, are much smoother and easily allow higher inter-element continuities. Kirchhoff-Love shell formulations are therefore very appealing for use in isogeometric frameworks. Besides avoiding the locking effects typically encountered in (low-order) Reissner-Mindlin elements [8], these isogeometric Kirchhoff-Love shells remove the need for rotational Degrees Of Freedom (DOFs). This not only yields a significant decrease of the resulting system matrix size, but also makes it dimensionally consistent, which leads to better condition numbers. Moreover, the geometrically exact character of the isogeometric approach can be of crucial advantage for shell models, which are known to be very sensitive to small imperfections [9]. A comprehensive overview of isogeometric shell formulations can be found in e.g. [10].

Although IGA has progressed fast since its conception little over a decade ago [1], it still suffers from some open issues - in particular when using a NURBS-based geometry, which is still the case for the vast majority of industrial CAD models. These are mainly due to the tensor-product topology of NURBS, which makes it difficult to generate free-form geometries with a single NURBS surface patch. Complex geometries therefore usually require the combining of multiple patches. Although $\mathrm{C}^{1}$-continuity is easily achieved within a single patch, obtaining it across patch boundaries is a different matter entirely - even maintaining $\mathrm{C}^{0}$-continuity can pose problems for arbitrary patch configurations. Given its importance in IGA, the problem of patch coupling has already been studied in many research papers - although often focused on $\mathrm{C}^{0}$-coupling [11-19]. Typically, mortar techniques [13-15], Nitsche's method [16-18], or penalty approaches [18] provide the coupling. In this context, it should be mentioned that the use of ReissnerMindlin formulations in isogeometric frameworks simplifies the issue of patch coupling, as those formulations only require $\mathrm{C}^{0}$-continuity. Examples of isogeometric multipatch Reissner-Mindlin shells can be found in e.g. [14, 20-22].

$\mathrm{C}^{1}$-coupling methods have been studied particularly in the context of Kirchhoff-Love shells [10, 23-30]. Maurin et al. [24] present a $\mathrm{C}^{1}$-coupling approach using the Rodrigues rotation matrix in an isogeometric framework for analysing periodic wave propagation. Kiendl et al. propose the bending strip method [25], which couples two bordering patches by adding a strip of fictitious material with a unidirectional bending stiffness. Schmidt et al. [26] employ this technique for coupling trimmed NURBS patches. This bending strip method is limited with regard to the nature of the interfaces it can treat without adding unwanted stiffening effects, and it can suffer from ill-conditioning. Goyal and Simeon modify the bending strip concept to remove its penalty parameter dependence and improve the resulting condition numbers, applying it to conforming meshes [27]. Duong et al. [10] enforce continuity across conforming Kirchhoff-Love patches through either a penalty formulation or the Lagrange multiplier method. Also studied by Apostolatos et al. [29] for non-conforming configurations, both these approaches enable the treatment of more arbitrary interface types but they require the proper choice of penalty parameters or 
Lagrange multiplier spaces. Also Nitsche's method requires a suitable choice of penaltylike stabilisation parameters. Guo and Ruess [30] use Nitsche's method for the coupling of Kirchhoff-Love shells, including non-conforming configurations and blended shell-solid models. Breitenberger et al. [31] also treat the coupling of non-conforming (and even trimmed) Kirchhoff-Love patches, but by means of a penalty approach.

This presence of non-conformities further complicates the coupling, but such configurations are very common in industrial CAD geometries. The modelling of complex shell structures therefore requires a framework that can impose $\mathrm{C}^{1}$-continuity of nonconforming patches in a flexible manner. The method introduced by the authors in [32] uses fundamental NURBS refinement properties to couple non-conforming patches in a robust manner. Although it mainly focuses on $\mathrm{C}^{0}$-problems [33], it also illustrates a simple method of collinear control variables for enforcing $\mathrm{C}^{1}$-continuity for relatively simple geometries.

The present work starts from the $\mathrm{C}^{0}$-coupling technique for non-conforming NURBS patches described in [32] for performing Kirchhoff-Love shell analysis of more arbitrary geometries. The result is a flexible and user-friendly coupling method which does not require the determining of proper penalty factors, nor the introducing of additional variables like Lagrange multipliers.

The remainder of the text is structured as follows. The next section briefly introduces some preliminaries on NURBS and their use in IGA. Section 3 then presents the proposed coupling method, detailing both the $\mathrm{C}^{0}$ - and the $\mathrm{C}^{1}$-coupling parts. Its accuracy is then illustrated through a set of numerical case studies in section 4 , followed by some concluding remarks.

\section{Preliminaries on NURBS-based isogeometric analysis}

This section gives a concise introduction to NURBS surfaces with their corresponding basis functions and briefly discusses their use in the context of IGA. More detailed information about IGA can be found in [1,2] and about NURBS in particular in [34].

NURBS can be constructed from B-splines. These are defined using a sequence of nondecreasing coordinates (or knots) in the parameter space $\xi$. A set of $n$ (one-dimensional) B-spline basis functions $N_{i}^{p}(\xi)(i=1, \ldots, n)$ can be generated recursively, using the Cox-de Boor recursion formula [35, 36], given a polynomial order $p$ and a knot vector $\boldsymbol{\Xi}=\left[\xi_{1}, \xi_{2}, \ldots, \xi_{n+p+1}\right]$ :

$$
\begin{array}{ll}
p=0: & N_{i}^{0}(\xi)= \begin{cases}1 & \xi_{i} \leq \xi<\xi_{i+1} \\
0 & \text { otherwise }\end{cases} \\
p>0: & N_{i}^{p}(\xi)=\frac{\xi-\xi_{i}}{\xi_{i+p}-\xi_{i}} N_{i}^{p-1}(\xi)+\frac{\xi_{i+p+1}-\xi}{\xi_{i+p+1}-\xi_{i+1}} N_{i+1}^{p-1}(\xi) .
\end{array}
$$

The use of $n$ B-spline basis functions requires the definition of $n+p+1$ knots $\xi_{k} \in \mathbb{R}$ $(k=1,2, \ldots, n+p+1)$.

Generating a NURBS basis from a B-spline one is done by adding a weight $w$ to each basis function. Characteristic of NURBS is the fact that multivariate bases are generated by taking the tensor product of univariate ones. Given two polynomial orders $p$ and $q$ with a corresponding pair of knot vectors $\boldsymbol{\Xi}=\left[\xi_{1}, \xi_{2}, \ldots, \xi_{n+p+1}\right]$ and 
$\boldsymbol{H}=\left[\eta_{1}, \eta_{2}, \ldots, \eta_{m+q+1}\right]$, a bivariate NURBS basis is defined as

$$
R_{i, j}^{p, q}(\xi, \eta)=\frac{N_{i}^{p}(\xi) M_{j}^{q}(\eta) w_{i, j}}{\sum_{i_{w}=1}^{n} \sum_{j_{w}=1}^{m} N_{i_{w}}^{p}(\xi) M_{j_{w}}^{q}(\eta) w_{i_{w}, j_{w}}} \quad \text { for } \begin{aligned}
& i=1, \ldots, n \\
& j=1, \ldots, m
\end{aligned}
$$

where $N_{i}^{p}(\xi)$ and $M_{j}^{q}(\eta)$ are univariate B-spline basis functions of order $p$ and $q$, associated with knot vectors $\boldsymbol{\Xi}$ and $\boldsymbol{H}$, respectively. A NURBS surface geometry $\boldsymbol{S}(\xi, \eta)$ is created by linearly combining the basis functions in a vectorial sense, using a set of vector-valued weighting coefficients $\mathbf{P}_{i, j} \in \mathbb{R}^{3}$ (called the control points, forming the control net):

$$
\mathbf{S}(\xi, \eta)=\sum_{i=1}^{n} \sum_{j=1}^{m} R_{i, j}^{p, q}(\xi, \eta) \mathbf{P}_{i, j} .
$$

Using an isoparametric approach, the basis functions making up a NURBS geometry can be used in shape function expansions for approximating field variables for that geometry. Let $u(\xi, \eta)$ be the primary variable. Its approximation $\hat{u}(\xi, \eta)$ can then be written as:

$$
u(\xi, \eta) \approx \hat{u}(\xi, \eta)=\sum_{i=1}^{n} \sum_{j=1}^{m} R_{i, j}^{p, q}(\xi, \eta) \cdot d_{i, j}=\sum_{l=1}^{n \cdot m} R_{l}(\xi, \eta) \cdot d_{l},
$$

where $d_{l}$ are the unknown control variables to be solved for, with $l=i+(j-1) \cdot m$ being a single counter replacing $i$ and $j$. Following a Galerkin approach, these same shape functions are then also used as test functions in a weighted residual formulation.

It is important to realise that eq. (3) describes a single NURBS surface patch. However, arbitrary free-form NURBS surfaces typically consist of multiple patches, which need to be coupled mathematically for analysis purposes. Doing so can be trivial in the case of $\mathrm{C}^{0}$-continuity (at least for conforming patches). However, for problems requiring $\mathrm{C}^{1}$-continuity the solution is not so straightforward. Ensuring a $\mathrm{C}^{1}$-coupling across (non-conforming) patch boundaries in a flexible manner is the subject of this paper; the following section explains the proposed approach, after a brief introduction to dynamic analysis of Kirchhoff-Love shells.

\section{Dynamic analysis of multipatch Kirchhoff-Love shells}

This work performs dynamic analysis on shell structures, modelled using isogeometric Kirchhoff-Love shells as introduced by Kiendl et al. [23]. Consider a thin shell of thickness $t$, and of a homogeneous, isotropic and linearly elastic material of density $\rho$, Young's modulus $E$ and Poisson's coefficient $\nu$. Let $\boldsymbol{X}=\left\{X_{1}, X_{2}, X_{3}\right\}^{T}$ denote the undeformed configuration in Cartesian coordinates in three-dimensional space. The shell deformation can be fully described by the displacement $\boldsymbol{u}=\left\{u_{1}, u_{2}, u_{3}\right\}^{T}$ of the midsurface. Assume now, for the purpose of illustration, that the shell is flat (i.e. a plate), with $X_{3}$ being the out-of-plane coordinate (such that $u_{3}$ represents the transverse displacement). Restricting the analysis to small deformations, the in-plane and out-of-plane motions of the flat geometry are governed by uncoupled dynamic equations. The steady-state dynamic bending motion in a thin flat shell (using a harmonic time-dependency $u(\boldsymbol{X}, t)=u(\boldsymbol{X}) \cdot \mathrm{e}^{\mathrm{j} \omega t}$ for a given angular frequency $\omega$ ) is governed by the following equation [37]:

$$
\nabla^{4} u_{3}\left(X_{1}, X_{2}\right)-k_{b}^{4} \cdot u_{3}\left(X_{1}, X_{2}\right)=\frac{p\left(X_{1}, X_{2}\right)}{D},
$$


with $\nabla^{4} \bullet=\frac{\partial^{4} \bullet}{\partial X_{1}^{4}}+\frac{\partial^{4} \bullet}{\partial X_{1}^{2} \partial X_{2}^{2}}+\frac{\partial^{4} \bullet}{\partial X_{2}^{4}}$ the biharmonic operator and $p\left(X_{1}, X_{2}\right)$ the external (normal) load. The bending stiffness $D$ and the bending wavenumber $k_{b}$ are defined as:

$$
\begin{aligned}
D & =\frac{E t^{3}}{12\left(1-\nu^{2}\right)}, \\
k_{b} & =\sqrt[4]{\frac{\rho t \omega^{2}}{D}} .
\end{aligned}
$$

The in-plane (membrane) motion is described by $u_{1}\left(X_{1}, X_{2}\right)$ and $u_{2}\left(X_{1}, X_{2}\right)$ and is governed by the following dynamic equations of motion (leaving out the $\left(X_{1}, X_{2}\right)$-dependency for the sake of brevity):

$$
\left\{\begin{array}{l}
\frac{\partial^{2} u_{1}}{\partial X_{1}^{2}}+\frac{1-\nu}{2} \frac{\partial^{2} u_{1}}{\partial X_{2}^{2}}+\frac{1+\nu}{2} \frac{\partial^{2} u_{2}}{\partial X_{1} \partial X_{2}}+\frac{1-\nu^{2}}{E} b_{X_{1}}+\frac{\rho\left(1-\nu^{2}\right) \omega^{2}}{E} u_{1}=0 \\
\frac{\partial^{2} u_{2}}{\partial X_{2}^{2}}+\frac{1-\nu}{2} \frac{\partial^{2} u_{2}}{\partial X_{1}^{2}}+\frac{1+\nu}{2} \frac{\partial^{2} u_{1}}{\partial X_{1} \partial X_{2}}+\frac{1-\nu^{2}}{E} b_{X_{2}}+\frac{\rho\left(1-\nu^{2}\right) \omega^{2}}{E} u_{2}=0
\end{array}\right.
$$

with $b_{X_{i}}$ the body force acting on the shell in direction $X_{i}$.

For shells, the bending and membrane phenomena are no longer decoupled. Although a shell formulation is not simply obtained by combining a plate and a membrane problem, describing the deformation as a function of convective curvilinear coordinates allows the stresses and strains in the curved shell to be separated into bending and membrane contributions. For the sake of brevity, the shell theory is not discussed in detail here, but more information concerning the employed Kirchhoff-Love shell formulation can be found in [23]. Because the present work studies dynamic, time-harmonic problems, the formulation in [23] needs to be extended to also take into account the inertia effects from eqs. (5) and (8) - resulting in a mass matrix in addition to the stiffness matrix. This is also done in [38].

Since eq. (8) is a set of second-order partial differential equations (PDEs), $\mathrm{C}^{0}$-continuous elements are required in a weighted residual formulation of the weak form. Equation (5), however, is a fourth order PDE, meaning that the shape function compatibility requirements demand at least $\mathrm{C}^{1}$-continuity. In conventional Lagrangian shell elements, with $\mathrm{C}^{0}$-continuity between them, this is typically ensured by introducing rotational DOFs. An isogeometric approach however, with higher inter-element continuity of the NURBS basis functions, allows the implementation of rotationless shells using only the translational displacements as DOFs. These are in this case given by the deformation $\boldsymbol{u}\left(\theta_{1}, \theta_{2}\right)$ in the global Cartesian coordinate system as a function of the (convective) curvilinear coordinates $\theta_{1}$ and $\theta_{2}$ of the undeformed shell surface $\mathbf{S}\left(\theta_{1}, \theta_{2}\right)$. The $\mathrm{C}^{1}$-continuity is embedded in the NURBS basis, provided that quadratic or higher-order functions are used. At least, this is the case within a single NURBS patch ${ }^{1}$, but across patch borders the $\mathrm{C}^{1}$ and even the $\mathrm{C}^{0}$-continuity should be explicitly enforced. The remainder of this section proposes a method for doing so in the general case of non-conforming meshes. First the need for imposing $\mathrm{C}^{0}$-continuity is discussed in section 3.1, then the additional enforcing of the required $\mathrm{C}^{1}$-continuity for the bending component, being the main focus of this work. After presenting in section 3.2 the technique for a two-patch configuration with

\footnotetext{
${ }^{1}$ This actually only holds true only if there are no repeated knots present that lead to $\mathrm{C}^{0}$-lines within the patch. If such lines are present, however, enforcing continuity across them can be done in very much the same way as for patch boundaries.
} 
a single interface, section 3.3 explains how it can be extended to a general multipatch configuration with an arbitrary number of interfaces and how the coupling constraints are imposed on the global system of equations.

\subsection{Enforcing $C^{0}$-continuity across a patch boundary}

For conforming meshes (i.e. meshes with matching mesh lines and the same interface basis functions), the $\mathrm{C}^{0}$-coupling across the interface is trivial and can be done by simply merging corresponding control variables on the interface (i.e. matching them one-to-one). This is not an option for non-conforming meshes, in which case the technique proposed in [32] is used, based on a master-slave formulation of coupling constraints. It is briefly summarised below.

Consider two non-conforming NURBS patches like in the top left illustration of fig. 1 . They are assumed to be of the same degree, which is a prerequisite for the method. If

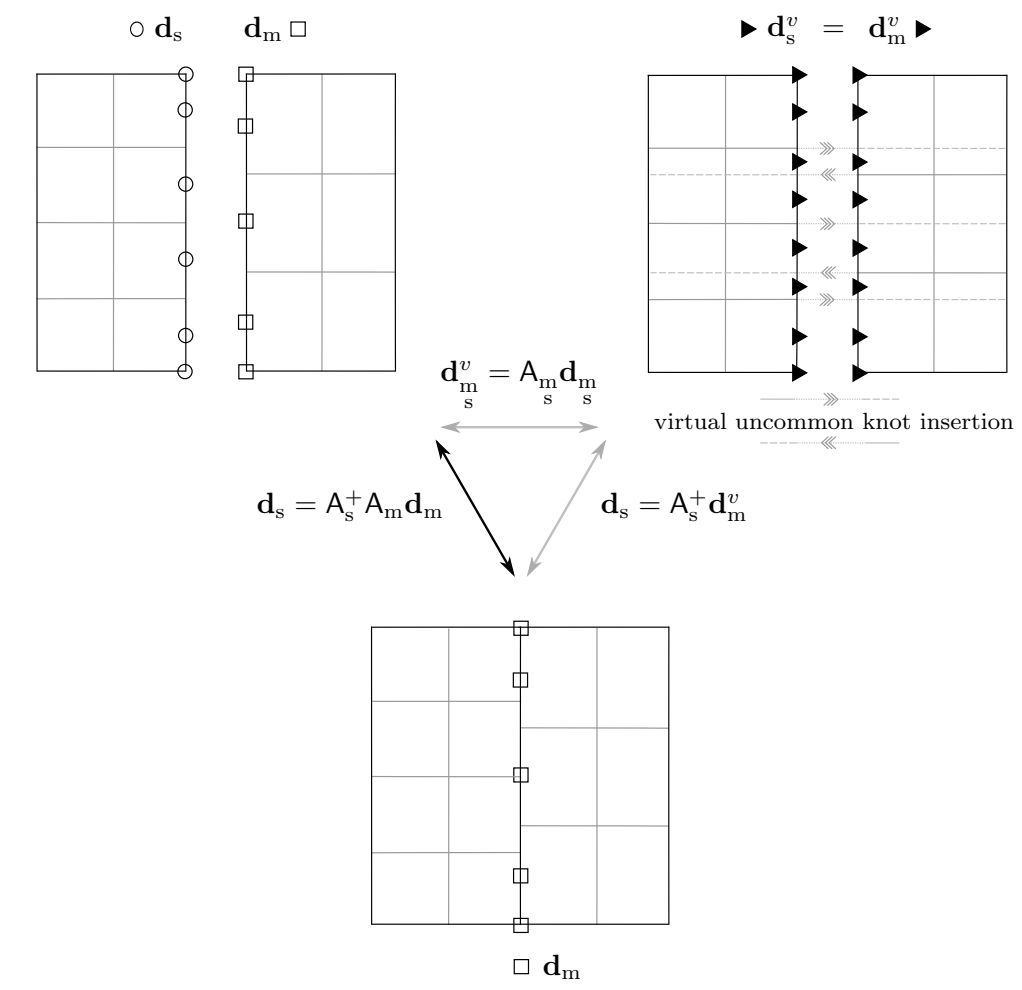

Figure 1: Illustration of the $\mathrm{C}^{0}$ coupling concept for non-conforming patches. Using restrictions resulting from the one-to-one matching of (virtually) uncommon knot-inserted meshes (top right) that are transformed back to the original control variables (top left), $\mathrm{C}^{0}$-continuity conditions can be (weakly) enforced within a master-slave relationship (bottom). The patch on the right acts as the master because it is the less refined one.

this is not the case, a straightforward workaround may be to order-elevate (one of) the patches until both are of the same degree. The two patches are coupled using a masterslave approach. Quantities belonging to the master and to the slave patch are indicated by subscripts ' $\mathrm{m}$ ' and ' $\mathrm{s}$ ', respectively - the choice of master and slave is at this point not important, but it is discussed further on in the text. For the coupling, both patches 
are virtually refined until they are conforming (like in the top right illustration), after which the regular one-to-one matching is enforced. This virtual refinement consists of inserting into each interface knot vector the knots that are present in the other patch's knot vector, but not in that of the patch itself (i.e. the uncommon knots). In other words: given the two interface knot vectors $\boldsymbol{\Xi}_{\mathrm{m}}$ and $\boldsymbol{\Xi}_{\mathrm{s}}$, refine them until they are both identical to $\boldsymbol{\Xi}_{\mathrm{m} \cup \mathrm{s}}=\boldsymbol{\Xi}_{\mathrm{m}} \cup \boldsymbol{\Xi}_{\mathrm{s}}$ (taking into account knot multiplicities). This procedure is referred to as virtual uncommon knot insertion. The refinement is virtual in the sense that the refined discretisations are never used for shape function expansions; they are merely used for obtaining the coupling equations resulting from a one-to-one matching of the refined control variables. It can be represented by extraction operators $A_{m}$ and $A_{s}$ that link the original control variables $\mathbf{d}_{\mathrm{m}}$ and $\mathbf{d}_{\mathrm{s}}$ to the control variables $\mathbf{d}_{\mathrm{m}}^{v}$ and $\mathbf{d}_{\mathrm{s}}^{v}$ of the virtually refined versions:

$$
\mathbf{d}_{\alpha}^{v}=\mathrm{A}_{\alpha} \mathbf{d}_{\alpha} \quad \alpha=\mathrm{m}, \mathrm{s} .
$$

These extraction operators $\mathrm{A}_{\alpha}$ express the linear combinations of the original control points that are needed for obtaining each refined control point. Details on how to compute them are given in [32], but it is worth remarking that their assembly requires only the interface knot vectors and NURBS weights, and no shape function evaluations whatsoever.

Figure 1 illustrates this coupling procedure. The bottom part of the figure represents the coupled patches (with $\mathbf{d}_{\mathrm{s}}$ and $\mathbf{d}_{\mathrm{m}}$ linked by eq. (11)), the top left part the uncoupled ones. The top right illustration shows the refined (virtual) configuration, where $\mathbf{d}_{1}^{v}=$ $\mathbf{d}_{2}^{v}$ has to hold. Ensuring the continuity thus comes down to enforcing $\mathbf{d}_{\mathrm{m}}^{v}=\mathbf{d}_{\mathrm{s}}^{v}$, or equivalently, using the original control variables $\mathbf{d}_{\alpha}$ :

$$
\mathrm{A}_{\mathrm{m}} \mathbf{d}_{\mathrm{m}}=\mathrm{A}_{\mathrm{s}} \mathbf{d}_{\mathrm{s}} .
$$

Directly enforcing this entire set of constraints would in general lead to an overconstrained interface and consequently converge to the wrong solution. The patches are instead coupled in a weak sense by expressing the variables of the slave patch in function of those of the master, yielding the following continuity relationship:

$$
\mathbf{d}_{\mathrm{s}}=\mathrm{A}_{\mathrm{s}}^{+} \mathrm{A}_{\mathrm{m}} \mathbf{d}_{\mathrm{m}},
$$

where $A_{s}^{+}$is the pseudo-inverse of $A_{s}$. This procedure reduces the number of interface constraints by satisfying eq. (10) in a least-squares sense, allowing an accurate global solution without overconstraining the model. For a proper choice of master and slave, the more coarsely discretised patch should act as the master. This way, the interface constraints can be satisfied in an approximate manner, using a smaller number of independent (and therefore possibly interfering) DOFs. Doing so sacrifices an exact fulfilment of interface constraints for a better global solution accuracy. The numerical examples in [32] confirm the good performance of this master-slave approach. Also, it is worth noting that in the special case of hierarchical meshes (where one patch is a refined version of the other one), this choice leads to exact $C^{0}$-continuity across the entire interface (since in that case $A_{s}$ is the identity matrix). Note that, since the total deformation is a vector field, eq. (11) should be imposed for the displacement in each of the three coordinate directions.

\subsection{Adding $C^{1}$-continuity for the out-of-plane displacement}

$\mathrm{C}^{0}$-continuity only involves the values of the shape functions on the interface and it is as such not strongly affected by the specific shape of the interface and the geometry around it. $\mathrm{C}^{1}$-continuity, however, relates to the shape function derivatives and it thus depends on the discretisation's behaviour around the interface and on the specific geometry. Care 
should therefore be taken to ensure that the coupling method can also properly handle less trivial patch configurations, such as patches meeting at an (out-of-plane) angle and/or with a curved interface.

Restricting the analysis to small deformations, the total displacement vector $\boldsymbol{u}$ at a given point on the midsurface can be decomposed into a locally out-of-plane (bending) component $u_{b}$ and a locally in-plane (membrane) component $u_{m}$, as fig. 2 illustrates. As explained above, it is the bending phenomenon that requires $\mathrm{C}^{1}$-continuity - the

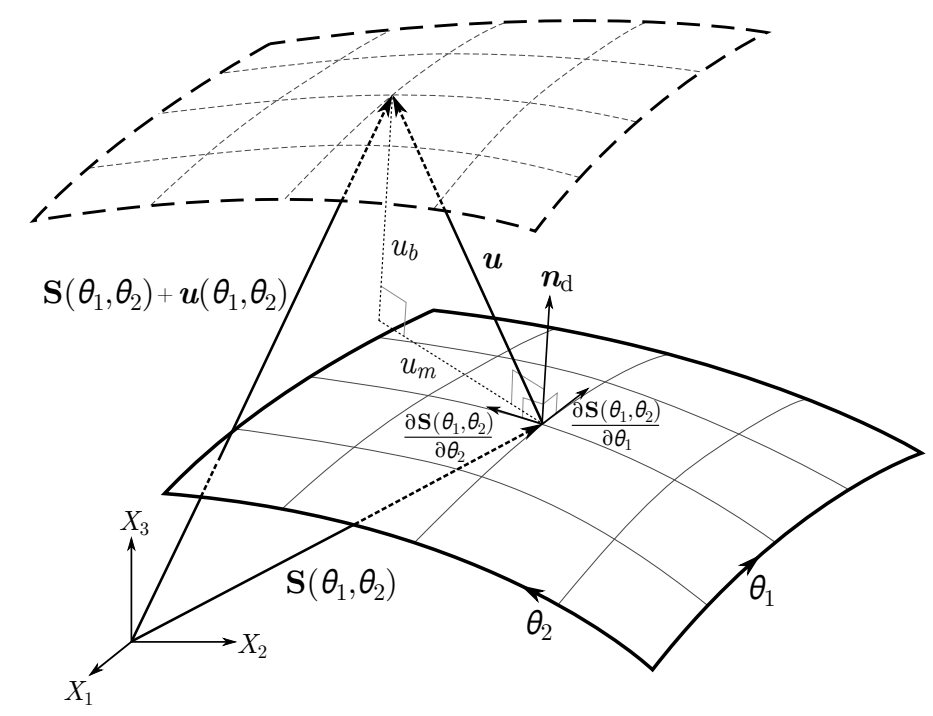

Figure 2: Decomposition of the (small) displacement $\boldsymbol{u}$ of the shell midsurface into a locally outof-plane component $u_{b}$ (normal to the shell midsurface) and a locally in-plane one $u_{m}$ (tangential to the shell midsurface). The bottom surface represents (a part of) the undeformed reference configuration $\mathbf{S}\left(\theta_{1}, \theta_{2}\right)$, the top one the corresponding deformed configuration.

in-plane displacement only needs a $\mathrm{C}^{0}$-continuous discretisation. The current section therefore proposes a method for the $\mathrm{C}^{1}$-coupling for only the bending component, i.e. the locally out-of-plane motion. In that sense, it should be noted that the proposed coupling is not a full $\mathrm{C}^{1}$-coupling, as the membrane deformation remains $\mathrm{C}^{0}$-continuous across the interface. The transverse displacement $u_{b}\left(\theta_{1}, \theta_{2}\right)$ can be defined as the displacement normal to the shell midsurface $\mathbf{S}\left(\theta_{1}, \theta_{2}\right)$, i.e. in the direction of the director vector $\boldsymbol{n}_{\mathrm{d}}\left(\theta_{1}, \theta_{2}\right)$. This vector is the unit normal vector of the (undeformed ${ }^{2}$ ) midsurface and can be calculated as follows (cf. fig. 2):

$$
\boldsymbol{n}_{\mathrm{d}}\left(\theta_{1}, \theta_{2}\right)=\frac{\frac{\partial \mathbf{S}\left(\theta_{1}, \theta_{2}\right)}{\partial \theta_{1}} \times \frac{\partial \mathbf{S}\left(\theta_{1}, \theta_{2}\right)}{\partial \theta_{2}}}{\left\|\frac{\partial \mathbf{S}\left(\theta_{1}, \theta_{2}\right)}{\partial \theta_{1}} \times \frac{\partial \mathbf{S}\left(\theta_{1}, \theta_{2}\right)}{\partial \theta_{2}}\right\|} .
$$

The out-of-plane component of displacement is then calculated as the dot product of the total displacement and the director vector:

$$
u_{b}\left(\theta_{1}, \theta_{2}\right)=\left\langle\boldsymbol{u}\left(\theta_{1}, \theta_{2}\right), \boldsymbol{n}_{\mathrm{d}}\left(\theta_{1}, \theta_{2}\right)\right\rangle,
$$

\footnotetext{
${ }^{2}$ Although the director vector in theory changes during deformation, the restriction to small deformations enables the use of the undeformed geometry as the reference configuration throughout the entire deformation.
} 
Generalising this, the out-of-plane or bending component $(\boldsymbol{\vee})_{b}$ of a vector quantity $\boldsymbol{}$ is analogously given as:

$$
(\bullet)_{b}=\left\langle\boldsymbol{\vee}\left(\theta_{1}, \theta_{2}\right), \boldsymbol{n}_{\mathrm{d}}\left(\theta_{1}, \theta_{2}\right)\right\rangle
$$

Now consider two NURBS patches $\mathbf{S}^{(i)}(\xi, \eta)$ with an arbitrary interface curve like in fig. 3a - this section uses the superscript $\bullet{ }^{(i)}$ for referring to quantities pertaining to patch $i$. The NURBS parametric coordinates $\xi$ and $\eta$ fulfil the role of curvilinear coordinates $\theta_{1}$ and $\theta_{2}$. For the sake of clarity, assume for now that the patches are conforming - the extension to non-conforming ones is analogous to the $\mathrm{C}^{0}$-coupling and is explained further on. Without loss of generality, the interface is taken to be located at $\eta=1$ for both patches. Along the interface the degree of continuity is already equal to that of the patch interior, but the proper continuity still needs to be enforced in the direction perpendicular to the interface. This direction can be different for each of the two patches $i$ forming the interface, and is given by the vector $\boldsymbol{n}_{\text {int }}^{(i)}(\xi)$, being the unit vector normal to the interface and tangential to the surface $\mathbf{S}^{(i)}$, pointing towards the interior of the patch (cf. fig. 3a). It can be calculated as a normalised vector product:

$$
\boldsymbol{n}_{\text {int }}^{(i)}(\xi)=\frac{\frac{\partial \mathbf{S}^{(i)}(\xi, 1)}{\partial \xi} \times \boldsymbol{n}_{\mathrm{d}}(\xi, 1)}{\left\|\frac{\partial \mathbf{S}^{(i)}(\xi, 1)}{\partial \xi} \times \boldsymbol{n}_{\mathrm{d}}(\xi, 1)\right\|}
$$

Note that these normal vectors are in general not parallel to the mesh lines - unless the latter are also normal to the interface.

As it is only the bending phenomenon that requires $\mathrm{C}^{1}$-continuity, the proposed coupling method aims to make the bending component of the corresponding directional derivative of the displacement continuous at the interface:

$$
\forall \xi: \quad\left(\frac{\partial \boldsymbol{u}^{(1)}(\xi, 1)}{\partial n_{\text {int }}^{(1)}}\right)_{b}+\left(\frac{\partial \boldsymbol{u}^{(2)}(\xi, 1)}{\partial n_{\text {int }}^{(2)}}\right)_{b}=0
$$

Writing out eq. (16) in terms of the deformation components in the Cartesian coordinate system and applying the definition of the bending component $(\boldsymbol{\nabla})_{b}$ then leads to the following equation to be enforced:

$$
\left\langle\left\{\frac{\partial u_{1}^{(1)}}{\partial n_{\mathrm{int}}^{(1)}}, \frac{\partial u_{2}^{(1)}}{\partial n_{\mathrm{int}}^{(1)}}, \frac{\partial u_{3}^{(1)}}{\partial n_{\mathrm{int}}^{(1)}}\right\}, \boldsymbol{n}_{\mathrm{d}}^{(1)}\right\rangle+\left\langle\left\{\frac{\partial u_{1}^{(2)}}{\partial n_{\mathrm{int}}^{(2)}}, \frac{\partial u_{2}^{(2)}}{\partial n_{\mathrm{int}}^{(2)}}, \frac{\partial u_{3}^{(2)}}{\partial n_{\mathrm{int}}^{(2)}}\right\}, \boldsymbol{n}_{\mathrm{d}}^{(2)}\right\rangle=0,
$$

with the dependency on $(\xi, 1)$ left out for the sake of brevity, and with the directional derivative $\frac{\partial \bullet}{\partial n_{\text {int }}}$ given by $\left\langle\boldsymbol{n}_{\text {int }}, \boldsymbol{\nabla} \bullet\right\rangle$. Note that when imposing eq. (17) along an interface, it is crucial that the director vector $\boldsymbol{n}_{\mathrm{d}}$ is consistently defined in both patches forming that interface. More specifically it should originate from the same side of the geometry, such that for a $\mathrm{G}^{1}$-continuous geometry the director vectors on the interface are identical in the neighbouring patches (rather than pointing in opposite directions). An interface should in this context be understood to be a line where any combination of two patches meet. Lines where three or more patches meet (cf. e.g. the honeycomb sandwich panel in section 4.4) therefore result in three or more separate interfaces. For each of those individual interfaces, a consistent director vector orientation should be used for coupling the involved patches. This does, however, not mean that the director vector should be 
consistently defined across all patches; this is not even possible in an arbitrary configuration.

Imposing constraint (17) is more complicated than enforcing the pure $\mathrm{C}^{0}$-continuity for the in-plane behaviour. The reason is that the shape function derivatives strongly depend on the specific geometrical mapping: derivatives on the interface are not only influenced by the interface control variables but also by those next to the interface, as fig. 3b illustrates. This prevents an elegant coupling that would require only the linking of control variables without the need for shape function evaluations - except in some special cases like the ones presented in [32]. Therefore, a collocation approach is chosen to enforce eq. (17) in a point-wise fashion. A proper choice of collocation points is

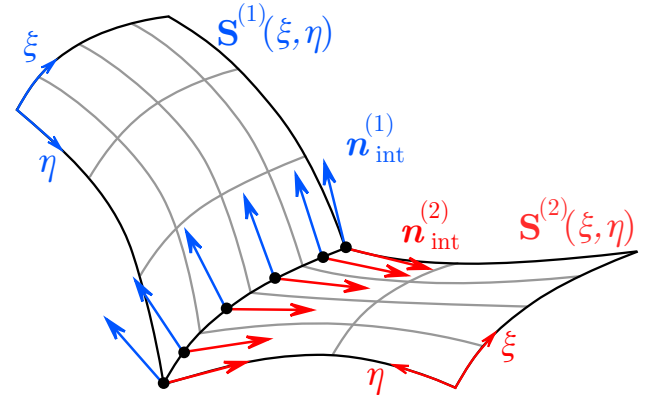

(a) The two NURBS surface $\mathbf{S}^{(i)}(\xi, \eta)$ with an indication of the collocation points $(\bullet)$ and the interface normals $\boldsymbol{n}_{\text {int }}^{(i)}$.

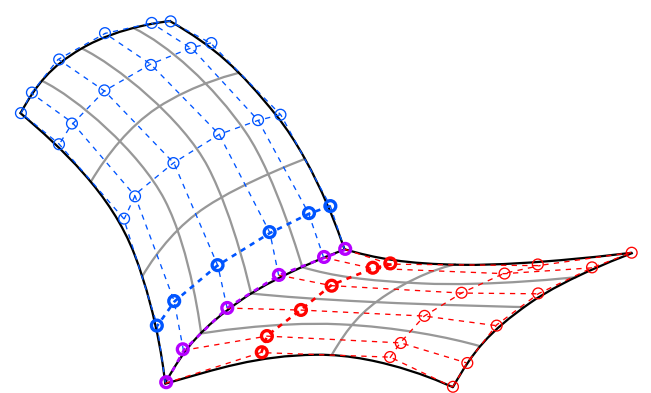

(b) Overlay of the mesh with the control polygon. The control variables indicated in bold determine the shape function derivatives at the interface.

Figure 3: A two-patch configuration illustrating the $\mathrm{C}^{1}$-coupling procedure. The grey lines delineate the elements.

of crucial importance with respect to the method's stability. The images of the Greville abscissae [39] are used as collocation points in this work. These abscissae are computed as knot averages, and they have already been applied successfully in isogeometric collocation frameworks [40, 41], with their interpolation proved to be stable (in 1D) up to at least polynomial order three [40]. There are also valid alternatives like Demko abscissae [42] or the variational collocation points proposed by Gomez and De Lorenzis [43]. The attractiveness of Greville abscissae lies partly in their easy computation. Given a knot vector $\left[\xi_{1}, \xi_{2}, \ldots, \xi_{n+p+1}\right]$, the $n$ Greville abscissae $\bar{\xi}_{j}$ are obtained as

$$
\bar{\xi}_{j}=\frac{1}{p}\left(\xi_{j+1}+\xi_{j+2}+\ldots+\xi_{j+p}\right), \quad j=1, \ldots, n .
$$

Collocating eq. (16) in (the images of) these Greville abscissae then yields

$$
\left(\frac{\partial \boldsymbol{u}^{(1)}\left(\bar{\xi}_{j}, 1\right)}{\partial n_{\text {int }}^{(1)}}\right)_{b}+\left(\frac{\partial \boldsymbol{u}^{(2)}\left(\bar{\xi}_{j}, 1\right)}{\partial n_{\text {int }}^{(2)}}\right)_{b}=0, \quad j=1, \ldots, n .
$$

Starting from the recast form (17) for the collocation and considering for each patch $i$ a shape function expansion (cf. eq. (4)) into a number $\left(L^{(i)}\right)$ of NURBS basis functions $R_{l, \tau}^{(i)}$ for $u_{\tau}(\tau=1,2,3)$, this can be rephrased in terms of the control variables (i.e. the 
DOFs) as follows:

$$
\sum_{\tau=1}^{3}\left(\sum_{l=1}^{L^{(1)}} C_{\tau, l, j}^{(1)} \cdot d_{\tau, l}^{(1)}+\sum_{l=1}^{L^{(2)}} C_{\tau, l, j}^{(2)} \cdot d_{\tau, l}^{(2)}\right)=0, \quad j=1, \ldots, n .
$$

The unknowns $d_{\tau, l}^{(i)}$ are the $L^{(i)}$ control variables in the shape function expansion for

the deformation component $u_{\tau}$ in patch $i$. Their corresponding coefficients $C_{\tau, l, j}^{(i)}$ are determined by both the shape function derivatives and the director vector components in the $j^{\text {th }}$ collocation point:

$$
C_{\tau, l, j}^{(i)}=\frac{\partial R_{l, \tau}^{(i)}\left(\bar{\xi}_{j}, 1\right)}{\partial n_{\mathrm{int}}^{(i)}} \cdot n_{\mathrm{d}, \tau}^{(i)}\left(\bar{\xi}_{j}, 1\right)=\left[\left\langle\boldsymbol{n}_{\mathrm{int}}^{(i)}, \nabla R_{l, \tau}^{(i)}\right\rangle n_{\mathrm{d}, \tau}^{(i)}\right]_{\left(\bar{\xi}_{j}, 1\right)}
$$

where $\bullet_{\tau}$ indicates the component of $\bullet$ in Cartesian coordinate direction $\tau$. A lot of these coefficients are equal to zero, since the shape function derivatives on the interface are determined only by the control variables belonging to shape functions on and next to the interface - as indicated in bold for the geometry control points in fig. 3b. Collecting these active control variables in separate vectors (with a consistent ordering) and denoting them as $\mathbf{d}_{\tau, \mathrm{O}}^{(i)}$ (On the interface) and $\mathbf{d}_{\tau, \mathrm{N}}^{(i)}$ (Next to the interface), the constraints in eq. (20) can be written in matrix form as follows:

$$
\sum_{\tau=1}^{3}\left(\mathrm{C}_{\tau, \mathrm{O}}^{(1)} \mathbf{d}_{\tau, \mathrm{O}}^{(1)}+\mathrm{C}_{\tau, \mathrm{N}}^{(1)} \mathbf{d}_{\tau, \mathrm{N}}^{(1)}+\mathrm{C}_{\tau, \mathrm{O}}^{(2)} \mathbf{d}_{\tau, \mathrm{O}}^{(2)}+\mathrm{C}_{\tau, \mathrm{N}}^{(2)} \mathbf{d}_{\tau, \mathrm{N}}^{(2)}\right)=\mathbf{0}
$$

where the $j^{\text {th }}$ row of the contribution matrices $\mathrm{C}_{\tau, \beta}^{(i)}$ (with $\beta=\mathrm{O}, \mathrm{N}$ ) contains the corresponding coefficients $C_{\tau, l, j}^{(i)}$ for the deformation in Cartesian coordinate direction $\tau$. Note that this equation enforces the continuity of the (bending component of the) first derivative of the displacement, but says nothing about the continuity of the displacement itself. For this conforming configuration, the approach explained in section 3.1 leads to $\mathbf{d}_{\tau, \mathrm{O}}^{(1)}=\mathbf{d}_{\tau, \mathrm{O}}^{(2)} \quad(\tau=1,2,3)$, guaranteeing (exact) $\mathrm{C}^{0}$-continuity of the total displacement field.

\section{Extension to non-conforming patches}

Imposing eq. (22) enforces the continuity of the gradient of the solution in the case of conforming meshes. For the extension to non-conforming meshes, a similar master-slave approach with virtual refinement as presented in section 3.1 is used. The $\mathrm{C}^{1}$-coupling should then be expressed for (the Greville abscissae of) the virtually refined configuration (cf. fig. 1). By choosing the master and the slave patch as before (denoted by subscripts ' $\mathrm{m}$ ' and 's', which replace the superscripts ' $\left.(i)^{\prime}\right)$, eq. (22) can then be written as:

$$
\sum_{\tau=1}^{3}\left(\mathrm{C}_{\tau, \mathrm{sO}}^{v} \mathbf{d}_{\tau, \mathrm{sO}}^{v}+\mathrm{C}_{\tau, \mathrm{sN}}^{v} \mathbf{d}_{\tau, \mathrm{sN}}^{v}+\mathrm{C}_{\tau, \mathrm{mO}}^{v} \mathbf{d}_{\tau, \mathrm{mO}}^{v}+\mathrm{C}_{\tau, \mathrm{mN}}^{v} \mathbf{d}_{\tau, \mathrm{mN}}^{v}\right)=\mathbf{0}
$$

where the superscript ' $v$ ' again indicates the virtually refined configuration. These constraints now need to be transformed back to the original DOFs. This can again be done by computing a set of extraction operators $A_{\alpha \beta}$ such that $\mathbf{d}_{\tau, \alpha \beta}^{v}=\mathrm{A}_{\alpha \beta} \mathbf{d}_{\tau, \alpha \beta}$ (cf. eq. (9)). The coupling constraints then become:

$$
\sum_{\tau=1}^{3}\left(\mathrm{C}_{\tau, \mathrm{sO}}^{v} \mathrm{~A}_{\mathrm{sO}} \mathbf{d}_{\tau, \mathrm{sO}}+\mathrm{C}_{\tau, \mathrm{sN}}^{v} \mathrm{~A}_{\mathrm{sN}} \mathbf{d}_{\tau, \mathrm{sN}}+\mathrm{C}_{\tau, \mathrm{mO}}^{v} \mathrm{~A}_{\mathrm{mO}} \mathbf{d}_{\tau, \mathrm{mO}}+\mathrm{C}_{\tau, \mathrm{mN}}^{v} \mathrm{~A}_{\mathrm{mN}} \mathbf{d}_{\tau, \mathrm{mN}}\right)=\mathbf{0}
$$


This equation can be simplified by setting $\mathrm{C}_{\tau, \alpha \beta}=\mathrm{C}_{\tau, \alpha \beta}^{v} \mathrm{~A}_{\alpha \beta}$. This is the mathematical equivalent of computing the contribution matrices in terms of the original interface DOFs $\mathbf{d}_{\tau, \alpha \beta}$ instead of in terms of the virtually refined ones $\mathbf{d}_{\tau, \alpha \beta}^{v}$. Indeed, rather than computing the contributions of the interface basis functions of the virtually refined configuration (i.e. $\mathrm{C}_{\tau, \alpha \beta}^{v}$ ) and then transforming them back to the original basis functions (using extraction operators $\mathrm{A}_{\alpha \beta}$ ), a more elegant approach is to directly calculate the contributions of the original interface basis functions (i.e. $\mathrm{C}_{\tau, \alpha \beta}$ ). In other words, eq. (24) can be obtained more efficiently by using the original patch discretisations evaluated in the Greville abscissae of the virtually refined configuration as collocation points. Equation (24) then becomes:

$$
\sum_{\tau=1}^{3}\left(\mathrm{C}_{\tau, \mathrm{sO}} \mathbf{d}_{\tau, \mathrm{sO}}+\mathrm{C}_{\tau, \mathrm{sN}} \mathbf{d}_{\tau, \mathrm{sN}}+\mathrm{C}_{\tau, \mathrm{mO}} \mathbf{d}_{\tau, \mathrm{mO}}+\mathrm{C}_{\tau, \mathrm{mN}} \mathbf{d}_{\tau, \mathrm{mN}}\right)=\mathbf{0}
$$

This direct use of the original patch discretisations prevents the need to calculate the extraction operators $\mathrm{A}_{\alpha \mathrm{N}}$ - the operators $\mathrm{A}_{\alpha \mathrm{O}}=\mathrm{A}_{\alpha}$ are anyway still required for the $\mathrm{C}^{0}$ coupling. Although eqs. (24) and (25) are mathematically equivalent, the latter allows a more efficient and more elegant implementation. It still uses the collocation points derived from the virtually refined configuration, but with the corresponding basis function contributions of the original discretisations.

Section 4.2.1 will illustrate that directly enforcing eq. (25) does not yield good results - overconstraining the interface in some locations. Therefore a least-squares approach is introduced, similarly to imposing eq. (11) instead of directly eq. (10) in the $\mathrm{C}^{0}$ part of the coupling. For an arbitrary curved shell structure, all slave DOFs $\mathbf{d}_{\tau, \mathrm{sN}}$ have some non-zero contributions $\mathrm{C}_{\tau, \mathrm{sN}}$ in eq. (25). Therefore, to take into account all these contributions, the entire equation is pre-multiplied with the pseudo-inverse matrix $\mathrm{R}_{\mathrm{sN}}=\left(\sum_{\tau=1}^{3} \mathrm{C}_{\tau, \mathrm{SN}}\right)^{+}$to introduce the master-slave formulation. Combining this with the $\mathrm{C}^{0}$-constraint $(11)$ in order to express the slave DOFs $\mathbf{d}_{\tau, \mathrm{sO}}$ as a function of the corresponding master DOFs $\mathbf{d}_{\tau, \mathrm{mO}}$, the following set of interface constraints is obtained:

$$
\left\{\begin{array}{l}
\mathbf{d}_{\tau, \mathrm{sO}}=\mathrm{R}_{\mathrm{SO}} \mathrm{A}_{\mathrm{m}} \mathbf{d}_{\tau, \mathrm{mO}} \quad \tau=1,2,3 \\
\mathrm{R}_{\mathrm{sN}} \sum_{\tau=1}^{3}\left[\mathrm{C}_{\tau, \mathrm{sN}} \mathbf{d}_{\tau, \mathrm{sN}}+\mathrm{C}_{\tau, \mathrm{mN}} \mathbf{d}_{\tau, \mathrm{mN}}+\left(\mathrm{C}_{\tau, \mathrm{sO}} \mathrm{R}_{\mathrm{SO}} \mathrm{A}_{\mathrm{m}}+\mathrm{C}_{\tau, \mathrm{mO}}\right) \mathbf{d}_{\tau, \mathrm{mO}}\right]=\mathbf{0} \\
\text { with } \quad \mathrm{R}_{\mathrm{sO}}=\mathrm{A}_{\mathrm{s}}^{+} \quad \text { and } \quad \mathrm{R}_{\mathrm{sN}}=\left(\sum_{\tau=1}^{3} \mathrm{C}_{\tau, \mathrm{sN}}\right)^{+}
\end{array}\right.
$$

This set of equations enforces $\mathrm{C}^{0}$-continuity of the entire deformation field and $\mathrm{C}^{1}$ continuity of the bending component. Several considerations arise when observing these coupling equations:

- The contribution matrices $\mathrm{C}_{\tau, \alpha \beta}$ are of dimension $n^{v} \times n_{\alpha}$, where $n_{\alpha}$ is the number of interface control points of the master or the slave patch $(\alpha=\mathrm{m}, \mathrm{s})$, and $n^{v}$ the corresponding one in the virtually refined configuration $\left(n_{\mathrm{s}}^{v}=n_{\mathrm{m}}^{v}=n^{v}=\right.$ $n$ ). The slave transformation matrices $\mathrm{R}_{\mathrm{SO}}$ and $\mathrm{R}_{\mathrm{sN}}$ both have dimensions $n_{\mathrm{s}} \times$ $n^{v}$. This means that eq. (26) represents $4 n_{\mathrm{s}}$ constraints, derived via the masterslave transformation matrices from the original $4 n^{v}$ constraints given by eqs. (10) and (25). 
- Because of the definition of the virtually refined configuration (cf. fig. 1), it always holds that $n_{\mathrm{s}} \leq n^{v}$, with the equality only valid for conforming or hierarchical interfaces. In that case, $R_{\mathrm{SO}}$ and $\mathrm{R}_{\mathrm{sN}}$ are square matrices of full rank; the premultiplication with $R_{\mathrm{SN}}$ then does not influence the coupling accuracy (and can thus even be left out) and $R_{s O}$ is even equal to the identity matrix: $R_{s O}=A_{s}=A_{m}=I_{n_{s}}$. For generally non-conforming meshes, $\mathrm{R}_{\mathrm{SO}}$ and $\mathrm{R}_{\mathrm{SN}}$ are rectangular (horizontal) matrices. Because this reduces the number of equations as compared to when the coupling restrictions (10) and (25) would be directly enforced, it prevents the interfaces from being overconstrained, as already investigated in more detail in [32].

- The extraction operators $\mathbf{A}_{\alpha}$ are independent of $\tau$, since it is assumed that the same NURBS expansion is used for each of the three deformation components $u_{\tau}$. More information about how to compute these matrices can be found in [32], but it involves only the interface knot vectors and NURBS weights.

- In the special case of a flat plate (say in the $X_{1} X_{2}$-plane) the in-plane DOFs $\mathbf{d}_{1, \alpha \beta}$ and $\mathbf{d}_{2, \alpha \beta}$ have no contributions in eq. (25): $C_{1, \alpha \beta}=C_{2, \alpha \beta}=\mathbf{0}$. The slave transformation matrix then reduces to $\mathrm{R}_{\mathrm{sN}}=\mathrm{C}_{3, \mathrm{sN}}^{+}$. Because of the linear independence of NURBS basis functions, $\mathrm{C}_{3, \mathrm{sN}}$ is of full column rank, such that $\mathrm{C}_{3, \mathrm{sN}}^{+} \mathrm{C}_{3, \mathrm{sN}}$ is equal to the identity matrix. The $\mathrm{C}^{1}$ part of eq. (26) then becomes:

$$
\mathbf{d}_{3, \mathrm{sN}}=-\mathrm{C}_{3, \mathrm{sN}}^{+}\left[\mathrm{C}_{3, \mathrm{mN}} \mathbf{d}_{3, \mathrm{mN}}+\left(\mathrm{C}_{3, \mathrm{sO}} \mathrm{R}_{\mathrm{sO}} \mathrm{A}_{\mathrm{m}}+\mathrm{C}_{3, \mathrm{mO}}\right) \mathbf{d}_{3, \mathrm{mO}}\right] .
$$

\subsection{Handling patch configurations with multiple interfaces and imposing the coupling} constraints

For geometries containing multiple interfaces, the coupling equations (26) need to be computed for each of them. Recall that an interface should be understood to be the meeting line of two patches. Lines where three or more patches meet (cf. e.g. the honeycomb sandwich panel in section 4.4) therefore result in three or more individual interfaces, which all yield their own coupling constraints. The resulting constraints pertaining to all the separate interfaces can be rewritten in homogeneous form and combined into a single matrix equation $R_{C} \mathbf{d}_{C}=\mathbf{0}$, where $R_{C}$ is the global constraints matrix and $\mathbf{d}_{C}$ the vector of all the unknowns involved in the coupling constraints (i.e. the coupling DOFs). In general this global constraints matrix $\mathrm{R}_{\mathrm{C}}$ contains linear dependencies (typically circular dependencies around interface cross points). For removing these dependencies and consequently solving the global system of equations while taking into account the resulting coupling constraints, the same approach as proposed in [32] is used; the remainder of this section briefly summarises it.

The linearly independent constraint equations (i.e. the linearly independent rows of $\mathrm{R}_{\mathrm{C}}$ ) are extracted by computing the rank-revealing $\mathrm{QR}$ factorisation of the transpose of $\mathrm{R}_{\mathrm{C}}$ [44]. For $n_{\mathrm{D}}$ linearly independent constraint equations, the resulting reduced constraints matrix $\mathrm{R}_{\mathrm{C}, \text { red }}$ is of size $n_{\mathrm{D}} \times\left(n_{\mathrm{D}}+n_{\mathrm{I}}\right)$. Based on this matrix, a set of $n_{\mathrm{D}}$ dependent coupling DOFs $\mathbf{d}_{\mathrm{D}}$ can be chosen - one for each remaining row. The set of $n_{\mathrm{I}}$ independent coupling DOFs is then $\mathbf{d}_{\mathrm{I}}=\mathbf{d}_{\mathrm{C}} \backslash \mathbf{d}_{\mathrm{D}}$. For numerical reasons, the coupling DOF corresponding to the entry with the largest absolute value in a given row is chosen as the dependent DOF for that row. ${ }^{3}$ Gaussian elimination is then applied to $\mathrm{R}_{\mathrm{C} \text {,red }}$, using

\footnotetext{
${ }^{3}$ For the rows that originate from a $\mathrm{C}^{0}$-constraint, this $\mathrm{DOF}$ is the row's slave DOF, due to the nature of the $\mathrm{C}^{0}$-coupling equations. For the rows relating to a $\mathrm{C}^{1}$-constraint this is not so readily determined - and these rows anyway do not in general involve only a single slave DOF.
} 
the entries for the dependent DOFs as pivots. The resulting matrix $R_{D I}$ still represents the original constraints: $R_{D I} d_{C}=\mathbf{0}$ is equivalent to $R_{C} d_{C}=\mathbf{0}$. Assume, without loss of generality, that the coupling DOFs are ordered in such a way that $\mathbf{d}_{\mathrm{C}}=\left\{\begin{array}{ll}\mathbf{d}_{\mathrm{D}}^{T} & \mathbf{d}_{\mathrm{I}}^{T}\end{array}\right\}^{T}$. The modified constraints matrix $\mathrm{R}_{\mathrm{DI}}$ can then be written as $\mathrm{R}_{\mathrm{DI}}=\left[\begin{array}{ll}\mathrm{I}_{n_{\mathrm{D}}} & -\mathrm{T}_{\mathrm{DI}}\end{array}\right]$, where $\mathrm{I}_{n_{\mathrm{D}}}$ is the identity matrix of size $n_{\mathrm{D}}$. The matrix $\mathrm{T}_{\mathrm{DI}}$ ( of dimensions $n_{\mathrm{D}} \times n_{\mathrm{I}}$ ) contains the global substitution relationships, expressing the interface coupling between the $n_{\mathrm{D}}$ dependent DOFs $\mathbf{d}_{\mathrm{D}}$ and the remaining independent DOFs $\mathbf{d}_{\mathrm{I}}$ :

$$
\mathbf{d}_{\mathrm{D}}=\mathrm{T}_{\mathrm{DI}} \mathbf{d}_{\mathrm{I}}
$$

By condensing out the DOFs $\mathbf{d}_{\mathrm{D}}$ from the system matrix according to eq. (28), the global system of equations can now be solved while taking into account the continuity conditions for all the interfaces.

It is worth noting here that due to the nature of the collocated $\mathrm{C}^{1}$-continuity requirements, the resulting coupling constraint equations can be numerically significantly less elegant than the pure $\mathrm{C}^{0}$ ones: they not only involve more DOFs, but there will likely also be some equations with large discrepancies between the smallest and the largest coefficient. Such discrepancies are undesirable from a numerical point-of-view, as they can significantly slow down the computation of $\mathrm{T}_{\mathrm{DI}}$ - which is otherwise negligible compared to system matrix assembly and solving - and also deteriorate the sparsity pattern of the resulting global system of equations. This can be partially remedied by setting entries in $R_{C}$ below a certain numerical threshold value to zero (cf. also [15]). Although the precise improvement will depend a.o. on the specific discretisation and on the number of interfaces at hand, doing this can lead to a significant computational speed-up without noticeably altering the accuracy above that threshold level.

\section{Numerical case studies}

This section presents some numerical examples to verify the accuracy of the proposed coupling method. First, an eigenvalue problem is studied for which an analytical solution can be used for benchmarking purposes. After that follow three boundary-value problems of increasing complexity to further assess the performance of the method. All isogeometric computations are carried out with in-house MATLAB code that uses functionalities from the IGAFEM framework [3].

\subsection{Eigenvalue problem for a simply supported rectangular plate}

Consider a rectangular plate (in the $x y$-plane) of width $L_{x}$ and height $L_{y}$ that is simply supported on all four sides. Studying only transversal (i.e. out-of-plane) deformations, its motion is governed by eq. (13). To a good approximation, the rotary inertia can be neglected for thin plates. The plate's eigenfrequencies $\omega_{m n \text {,ex }}$ and corresponding eigenmodes $u_{m n, \mathrm{ex}}(x, y)$ then have analytical expressions [45]:

$$
\begin{array}{ll}
\omega_{m n, \mathrm{ex}}=\pi^{2} \sqrt{\frac{D}{\rho t}}\left(\frac{m^{2}}{L_{x}^{2}}+\frac{n^{2}}{L_{y}^{2}}\right), & m, n=1,2, \ldots, \\
u_{m n, \mathrm{ex}}(x, y)=\sin \frac{m \pi x}{L_{x}} \sin \frac{n \pi y}{L_{y}}, & m, n=1,2, \ldots
\end{array}
$$

The properties of the plate are chosen as follows: $L_{x}=1 \mathrm{~m}, L_{y}=0.5 \mathrm{~m}, t=5 \mathrm{~mm}$, $E=200 \mathrm{GPa}, \nu=0.3$ and $\rho=7800 \mathrm{~kg} / \mathrm{m}^{3}$. The eigenfrequencies are then numerically 
computed using three different multipatch meshes, as shown by fig. 4. The meshes contain eight B-spline patches, in fig. 4a in a conforming configuration ('MP-C') and in figs. 4b and $4 \mathrm{c}$ in a non-conforming one ('MP-NC(-CI)'). Figure 4c in addition also has curved interfaces, adding to the difficulty of properly coupling the patches in a $\mathrm{C}^{1}$-sense. All configurations have about the same average mesh size and are studied using B-spline basis functions of varying polynomial order $p$. The average mesh size $h$ is calculated as $h=\sqrt{\frac{L_{x} \cdot L_{y}}{N_{\mathrm{el}}}}$ with $N_{\mathrm{el}}$ the total number of elements.

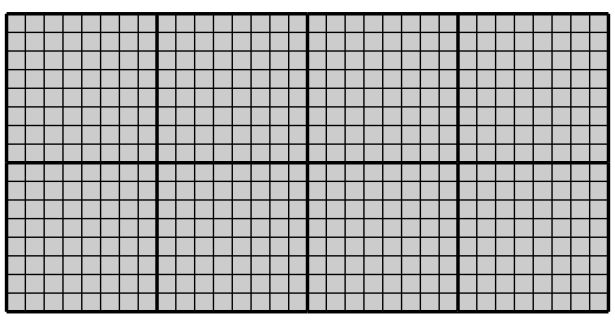

(a) Eight conforming patches ('MP-C'), with $N_{\mathrm{el}}=512$ and $h=0.031 \mathrm{~m}$.

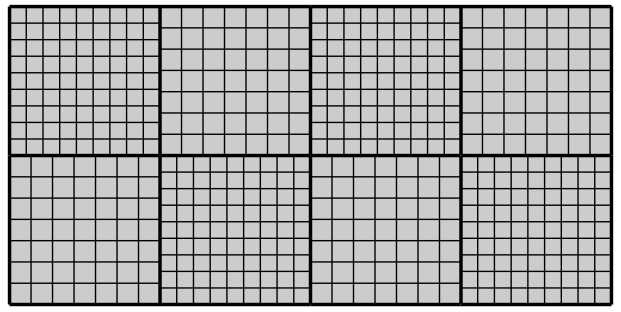

(b) Eight non-conforming patches ('MP-NC'), with $N_{\mathrm{el}}=520$ and $h=0.031 \mathrm{~m}$.

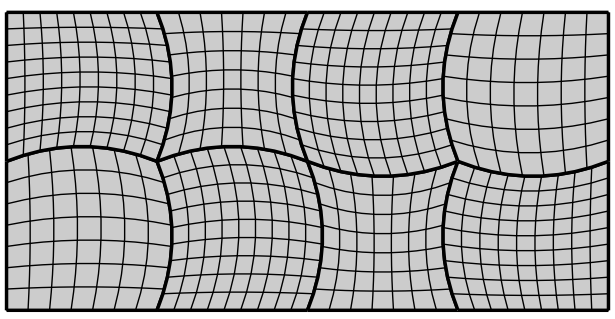

(c) Eight non-conforming patches with curved interfaces ('MP-CI-NC'), with $N_{\mathrm{el}}=$ 520 and $h=0.031 \mathrm{~m}$.

Figure 4: The conforming and non-conforming multipatch meshes used for solving the eigenvalue problem for the simply supported plate. The thicker lines are the patch boundaries.

The eigenfrequency spectrum of the models is investigated by plotting the relative error of the numerically obtained eigenfrequencies $\hat{\omega}_{i}$ (sorted by increasing magnitude) versus the normalised mode index $i / \mathrm{N}$. The number $\mathrm{N}$ represents the total number of modes (or DOFs) present in the model. Figure 5 plots these eigenfrequency error spectra for the different models using quadratic and cubic basis functions. As a benchmark curve, the errors for the single patch model ('SP') with the same elements as in fig. 4a are also 


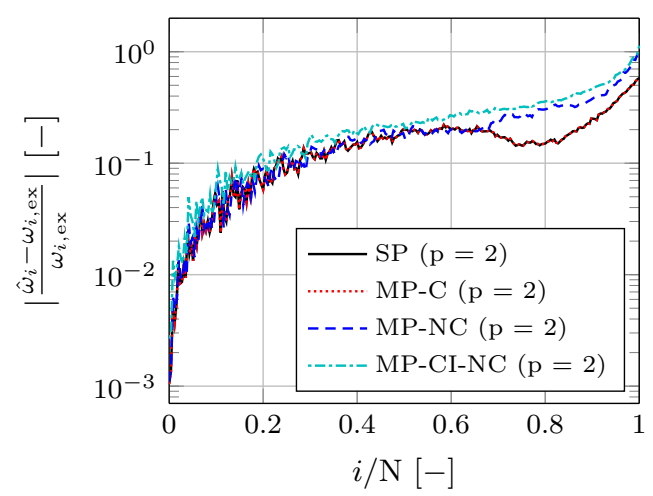

(a) Using quadratic B-spline basis functions.

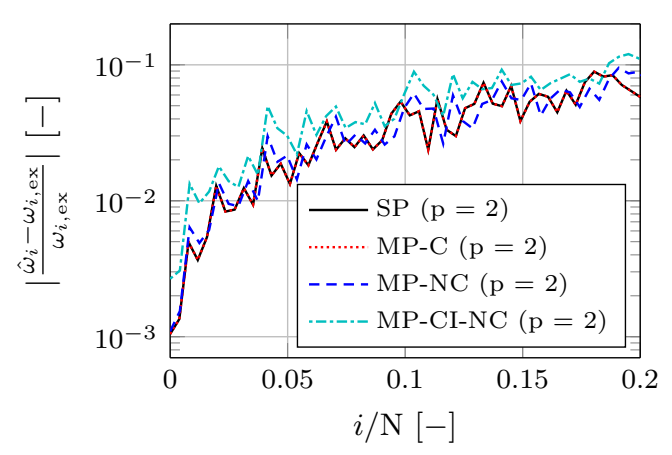

(c) A close-up of the lower modes of (a).

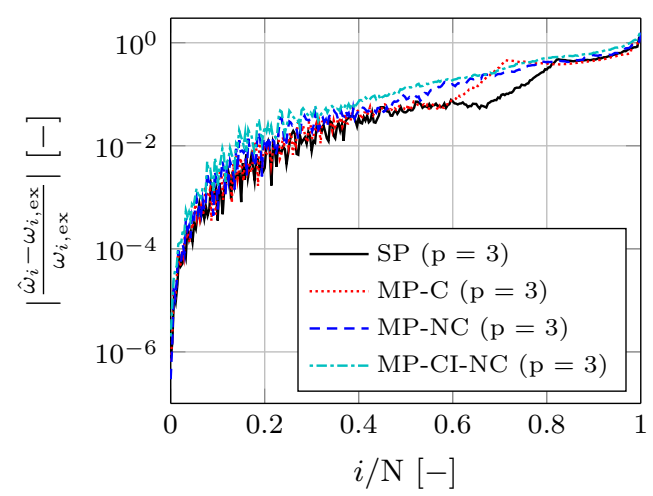

(b) Using cubic B-spline basis functions.

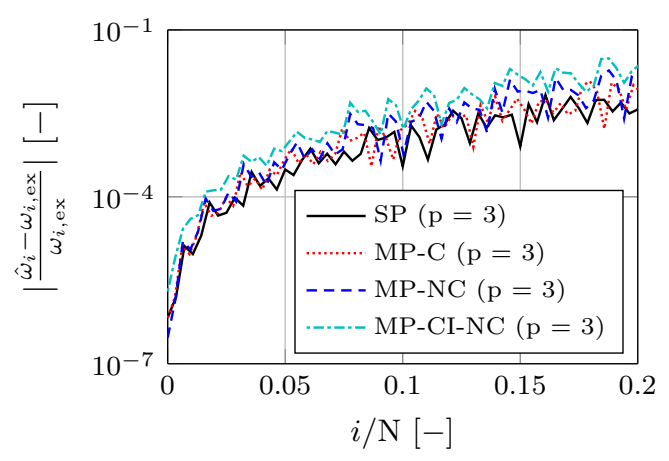

(d) A close-up of the lower modes of (b).

Figure 5: Relative error of the eigenfrequencies versus the normalised mode index for the simply supported rectangular plate, comparing the meshes from fig. 4 to the corresponding single patch solution ('SP'). The multipatch models perform very similarly to the single patch, in particular in the lower part of the spectrum.

shown. The figure presents several observations. First of all, the higher-frequency part of the spectrum does not yield accurate results; as the normalised mode index increases, the errors on the eigenfrequencies (and -modes) become increasingly larger. This has been investigated in more detail in other works [46-48]. But more important in the context of this work is that the accuracy of the multipatch models is very similar to the single patch one. The error for the conforming multipatch configuration even coincides (up to machine precision) with its single patch equivalent in the quadratic case. This illustrates the very accurate $\mathrm{C}^{1}$-coupling in the MP-C model, as the quadratic single patch model is only $\mathrm{C}^{1}$-continuous across its knots. The cubic MP-C model, lacking $\mathrm{C}^{2}$-continuity across its patch boundaries, also performs very similarly but not identically to the single patch solution, which does have $\mathrm{C}^{2}$-continuity everywhere. Also the non-conforming models yield nearly the same eigenfrequency errors as the single patch solution, in particular in the lower (still accurate) part of the spectrum. The accuracy of the models with curved interfaces (MP-CI-NC) is only very slightly lower, which is partly due to its less uniform mesh size. As expected, the cubic models are all significantly more accurate than their quadratic counterparts. 
Figure 6 studies in more detail the performance for the first two eigenmodes (i.e. with $(m, n)=(1,1)$ for the first and $(m, n)=(2,1)$ for the second mode). Using models of the same mesh topologies as in fig. $4^{4}$, it plots the convergence of the relative error on the eigenfrequency and on the mode shape for these modes. For the mode shapes, the $L^{2}$ relative error $\left\|\hat{u}_{m n}-u_{m n, \mathrm{ex}}\right\|_{2} /\left\|u_{m n, \mathrm{ex}}\right\|_{2}$ is used as error criterion, with the $L^{2}$ norm defined as $\|\bullet\|_{2}=\sqrt{\int_{\Omega} \bullet 2 \mathrm{~d} \Omega}$. The curves show that all the models converge with decreasing mesh size, for the frequencies as well as for the mode shapes. As indicated by the slope triangles, the theoretical convergence rates (cf. $[46,49])$ are obtained for the different polynomial orders. Interesting to note is that the conforming multipatch models perform identically to the single patch models in the quadratic and even in the cubic

${ }^{4}$ All the studied non-conforming models here are also non-hierarchical.

$$
\begin{aligned}
& \rightarrow \mathrm{SP}(\mathrm{p}=2) \quad \multimap-\mathrm{MP}-\mathrm{C}(\mathrm{p}=2) \quad \square \text { MP-NC }(\mathrm{p}=2) \quad \triangle \text { MP-CI-NC }(\mathrm{p}=2) \\
& -x-\text { SP }(\mathrm{p}=3) \quad-0-\text { MP-C }(\mathrm{p}=3) \quad \text { - 七- MP-NC }(\mathrm{p}=3) \quad-\triangle-\text { MP-CI-NC }(\mathrm{p}=3)
\end{aligned}
$$

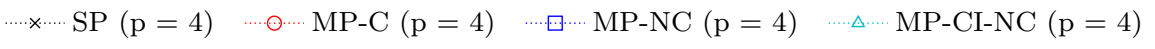
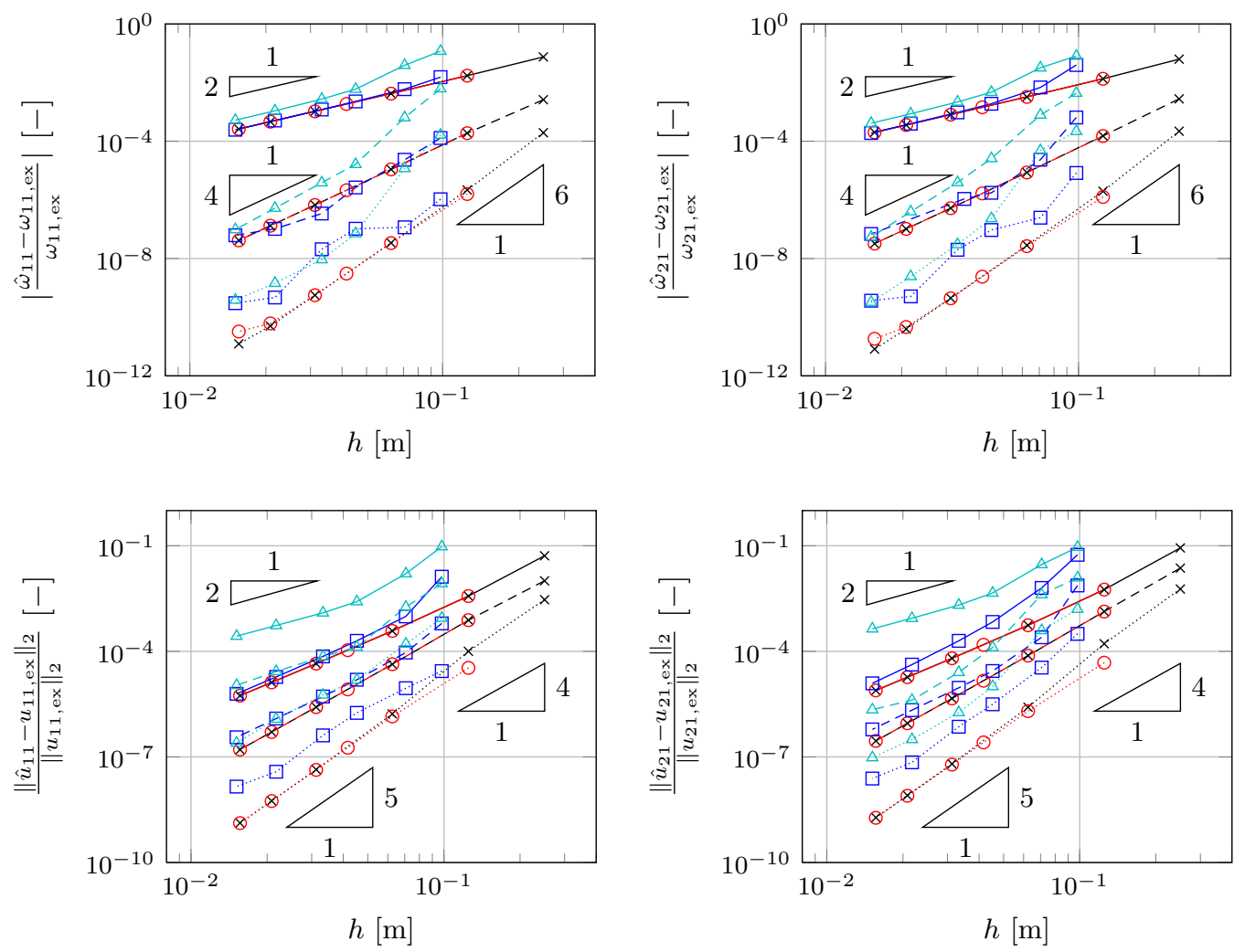

(a) First mode $(m=1, n=1)$.

(b) Second mode $(m=2, n=1)$.

Figure 6: Convergence of the relative error versus (average) mesh size $h$ for the first two eigenfrequencies (top) and the corresponding mode shapes (bottom) of the rectangular plate, for quadratic, cubic and quartic discretisations. 
case, indicating again a proper coupling across patch boundaries. Also for the quartic discretisations the performance is almost identical. The quadratic and cubic MP-NC models perform very similarly to the single patch benchmark as well, in particular for the eigenfrequencies. The accuracy of the MP-CI-NC models is lower, especially for the mode shapes. This is partially due to the irregularity of the meshes (containing curved elements of non-uniform size) which causes a vertical shift of the error curves. But their convergence rates also decrease with respect to their single patch counterparts; these slightly non-optimal slopes possibly indicate that the collocation of the $\mathrm{C}^{1}$-constraints does not perform as well for arbitrary meshes as for regular ones. The higher-order models also seem to suffer more from this effect than the lower-order ones. Regardless of the slightly non-optimal convergence in this case, however, the MP-CI-NC curves still clearly and consistently converge.

\subsection{Scordelis-Lo roof}

This section studies the Scordelis-Lo roof benchmark, a part of the well-known shell obstacle course proposed by Belytschko et al. [50]. It is a static problem, consisting of a simply supported cylindrical shell section uniformly loaded by gravity, as described in fig. 7a. The benchmark value is the vertical $(z$-)displacement of the midpoint of the

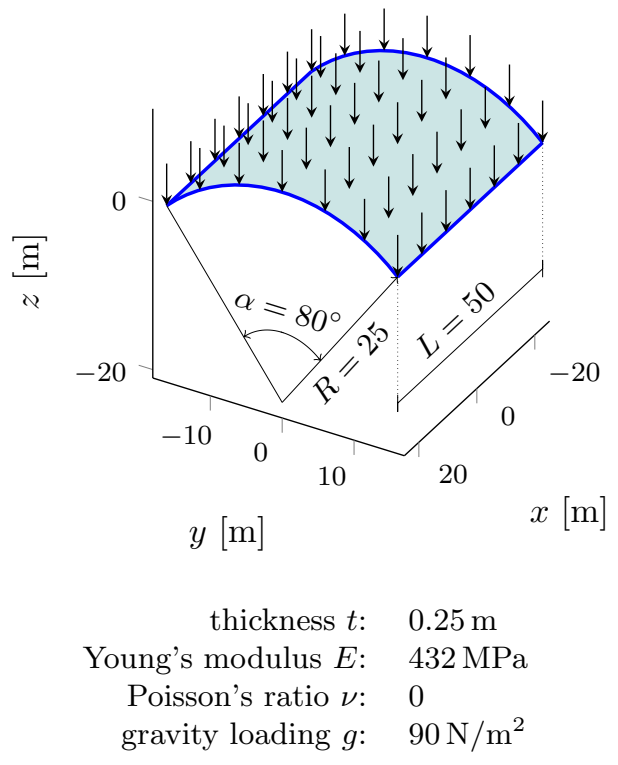

(a) Problem setting, consisting of a cylindrical section loaded uniformly by gravity. The straight edges are free, while the curved edges can only move in $x$-direction.

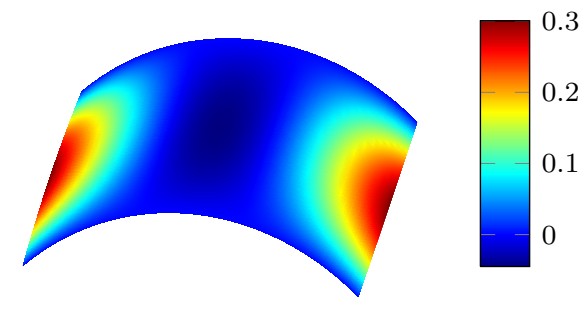

(b) Reference solution (calculated with a quintic single patch model of 29400 elements), showing a colour plot of the vertical displacement $[\mathrm{m}]$ in the negative $z$-direction.

Figure 7: The Scordelis-Lo roof benchmark problem description. All numerical values are given in SI units.

straight side edges. As already discussed in previous works [23, 32], the reference displacement of $0.3024 \mathrm{~m}$ as given by [50] differs slightly from the one obtained when using isogeometric shells (i.e. a displacement of $0.3006 \mathrm{~m}$ ). Therefore, a very fine single patch model (intrinsically meeting the continuity requirements) is used as reference solution here; in 
particular one consisting of 29400 quintic NURBS elements, yielding just under 100000 reference DOFs. The reference benchmark displacement then becomes $0.300592457 \mathrm{~m}$. Figure $7 \mathrm{~b}$ shows a colour plot of the reference vertical displacement field.

This problem is modelled using three different types of meshes (cf. fig. 8), much the same as in the previous section: a single patch ('SP'), a conforming four-patch ('MP$\mathrm{C}$ '), and a corresponding non-conforming model ('MP-NC'). The multipatch models are asymmetric, with the interfaces positioned off-centre. Using these mesh configurations,

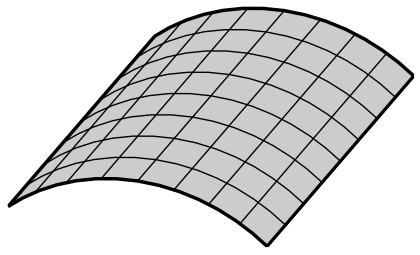

(a) Single patch mesh ('SP').

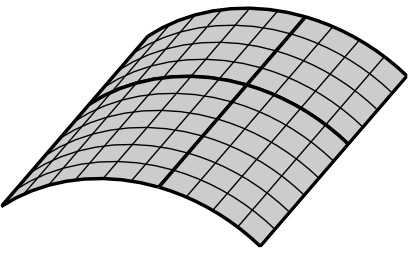

(b) Conforming four-patch mesh ('MP-C') with the interfaces placed off-centre, yielding an asymmetric patch configuration.

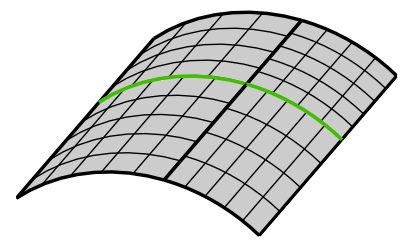

(c) Non-conforming fourpatch mesh ('MP-NC'), with the same interfaces as in (b). The green (curved) interfaces are studied in more detail in section 4.2.1.

Figure 8: The topology of the different meshes used for studying the Scordelis-Lo benchmark problem. The thicker lines are the patch boundaries.

the convergence behaviour is studied for different polynomial orders $p$, from quadratic to quartic. Figure 9 shows convergence curves for the relative error of the benchmark displacement ${ }^{5}$ versus the average mesh size $h$. The plots also show the theoretical slope markers for the $L^{2}$ error (cf. $[46,49]$ ) for the reader's convenience. These would indicate that the single patch and the conforming models are superconvergent; note, however, that the error concerns a single point here (as according to the original benchmark definition) rather than an integral $L^{2}$ error. Based on heuristic findings, one might expect that both errors converge at the same rate, but there is no theoretical support for this to the authors' knowledge. One should therefore be careful when drawing conclusions based on these slope indicators. For all polynomial orders, the conforming models perform nearly identically to the single patch equivalents. In the quadratic case, where the single patch models do not possess higher continuity than the multipatch ones, even the nonconforming meshes match the single patch performance. Although the cubic and quartic non-conforming models follow the conforming ones for the more coarse meshes, their convergence slows down as the mesh size decreases (and approaches the fine limit where the error magnitude reaches that of the reference solution). Similar discrepancies in performance between conforming and non-conforming models can be observed for models of even higher order (e.g. fifth- or sixth-order, which are not shown here). Nonetheless, despite this non-optimal convergence, the non-conforming models still clearly converge.

It is not only the enforcing of the bending's $\mathrm{C}^{1}$-continuity itself that is crucial, but also the way the $\mathrm{C}^{1}$ coupling restrictions are taken into account is of great importance, as illustrated in the remainder of this case study.

\footnotetext{
${ }^{5}$ Given the asymmetry of the model, the average of the two straight-edge midpoint displacements is used as the benchmark here.
} 


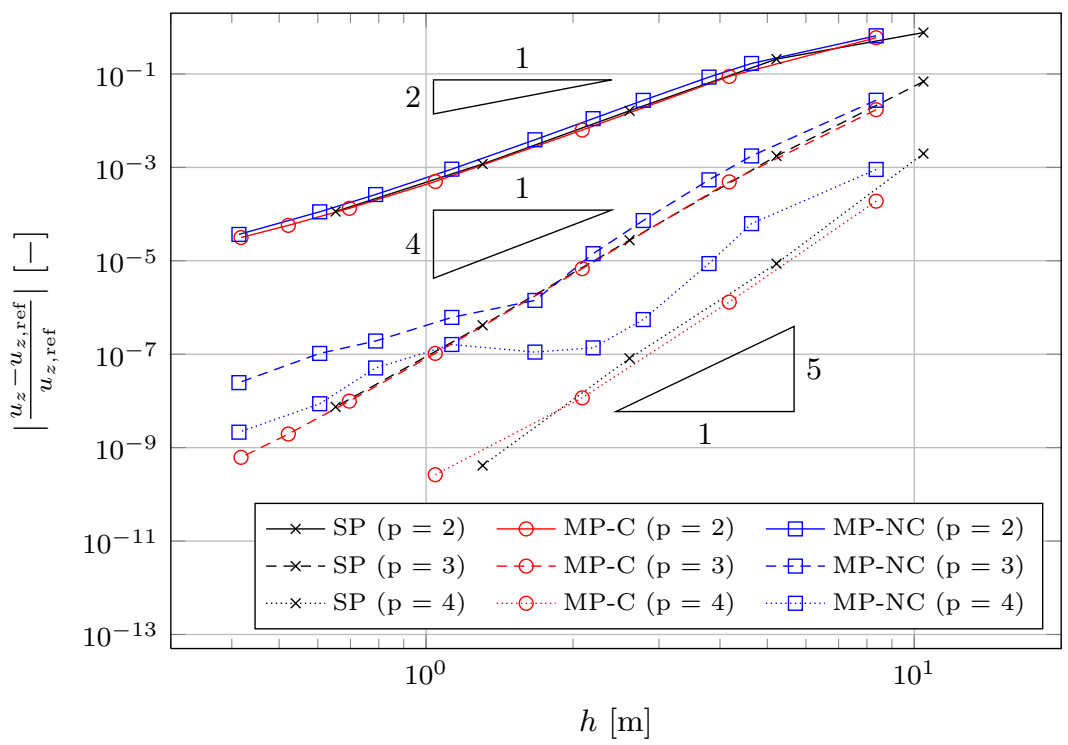

Figure 9: Convergence of the relative error of the benchmark displacement versus the average mesh size $h$.

\subsubsection{On the importance of using a master-slave formulation}

To study more in detail the importance of a proper $\mathrm{C}^{1}$-coupling for the bending motion, and of the master-slave transformation introduced by the matrices $R_{S O}$ and $R_{s N}$, four additional sets of model variants are studied:

$\mathbf{C}^{0} \mathbf{S M} / \mathbf{C}^{1} \mathbf{S M}$ : Models almost identical to the proposed approach, except that the more refined patch serves as the master instead of the slave. In this sense, it is the opposite master-slave formulation of the proposed method.

$\mathbf{C}^{0} \mathbf{M S} / \mathbf{C}^{1} \mathbf{D}$ : Models where both the $\mathrm{C}^{0}$-continuity of the full displacement field and the $\mathrm{C}^{1}$-continuity of the bending component are imposed, but using a direct approach instead of a master-slave framework for the latter. This means that eq. (25) is used directly to enforce the bending motion's $\mathrm{C}^{1}$-continuity, instead of using the lower part of eq. (26). Both sets of equations are derived from the same conceptual restrictions, but eq. (26) applies a master-slave transformation $\mathrm{R}_{\mathrm{sN}}$ whereas eq. (25) does not.

$\mathbf{C}^{0} \mathbf{D} / \mathbf{C}^{1} \mathbf{D}$ : Models where both the $\mathrm{C}^{0}$-continuity of the full displacement field and the $\mathrm{C}^{1}$-continuity of the bending component are imposed, but using a direct approach instead of a master-slave framework. This means that instead of using eq. (26) for condensing out the dependent DOFs, eqs. (10) and (25) are used to enforce $\mathrm{C}^{0}$-continuity and $\mathrm{C}^{1}$-continuity, respectively - without the master-slave transformation matrices $\mathrm{R}_{\mathrm{sO}}$ and $\mathrm{R}_{\mathrm{sN}}$.

The proposed approach is referred to here as ' $\mathrm{C}^{0} \mathrm{MS} / \mathrm{C}^{1} \mathrm{MS}$ '. All these variants are computed for both conforming and non-conforming meshes of third order - except for the $\mathrm{C}^{0} \mathrm{SM} / \mathrm{C}^{1} \mathrm{SM}$ variant which is identical to the proposed approach in the case of conforming configurations. Figure 10 presents the corresponding convergence curves for the 


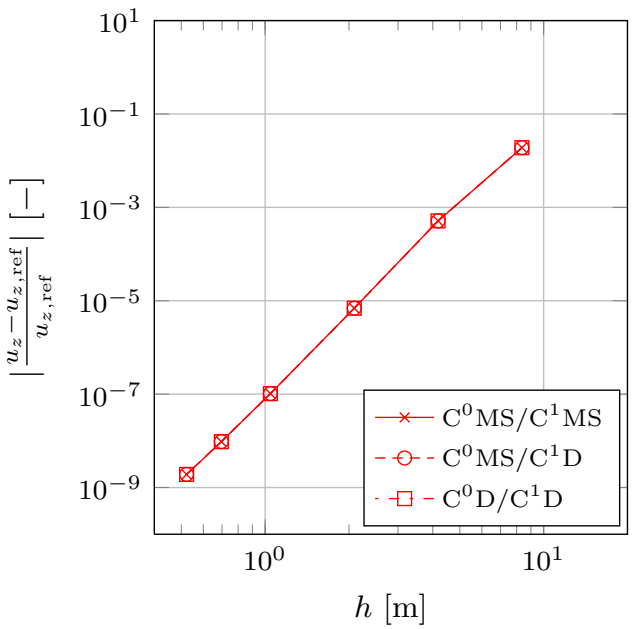

(a) Using conforming meshes (cf. fig. 8b).

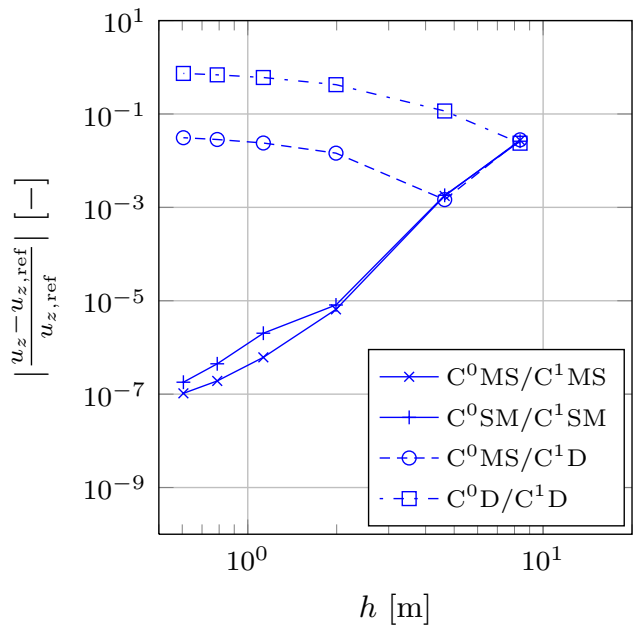

(b) Using non-conforming meshes (cf. fig. 8c).

Figure 10: Convergence of the relative error of the benchmark displacement versus the average mesh size $h$, using different coupling method variants. All solutions are computed using a cubic NURBS basis $(\mathrm{p}=3)$.

benchmark displacement. Figure 10a shows that for conforming meshes there is no distinction between the different model variants. The master-slave formulation does not introduce added value because all matrices $\mathrm{A}_{\alpha}$ are identity matrices. However, for nonconforming configurations the master-slave formulation is crucial. Only the $\mathrm{C}^{0} \mathrm{MS} / \mathrm{C}^{1} \mathrm{MS}$ and $\mathrm{C}^{0} \mathrm{SM} / \mathrm{C}^{1} \mathrm{SM}$ models converge; the direct approach causes stagnation at low accuracy levels. It overconstrains the solution around the interfaces, sacrificing global accuracy for continuity. Enforcing only the $\mathrm{C}^{1}$ restrictions in a direct sense $\left(\mathrm{C}^{0} \mathrm{MS} / \mathrm{C}^{1} \mathrm{D}\right)$ is slightly more accurate but still does not converge. Although there is only a very small performance difference between the two master-slave models, the proposed $\mathrm{C}^{0} \mathrm{MS} / \mathrm{C}^{1} \mathrm{MS}$ variant does slightly better than the $\mathrm{C}^{0} \mathrm{SM} / \mathrm{C}^{1} \mathrm{SM}$ alternative.

To better understand these observations, it is interesting to look at the behaviour of the different models around the interfaces. This is done for the (cubic) non-conforming mesh shown in fig. 8c, which corresponds to an average element size $h=4.64 \mathrm{~m}$ (i.e. the second most coarse data points in fig. 10b). Figure 11 plots the different solutions evaluated at the green interfaces lines in fig. $8 \mathrm{c}$ versus the $y$-coordinate along the interface. Figure 11a shows the total displacement $u_{\text {tot }}=\sqrt{u_{x}^{2}+u_{y}^{2}+u_{z}^{2}}$, while fig. 11b plots the bending component of the displacement derivative (cf. eq. (14)) in the $x$-direction (i.e. normal to the interface). The plots compare these entities evaluated in patches on either side of the interface to the (single patch) reference solution. The dotted vertical line at $y_{\times}$marks the cross point where the green and black interfaces in fig. $8 \mathrm{c}$ meet.

These curves show that only the models where the full coupling is incorporated using a master-slave formulation obtain a correct interface behaviour - at least qualitatively. For the other models, the following can be observed:

- The $\mathrm{C}^{0} \mathrm{MS} / \mathrm{C}^{1} \mathrm{D}$ model produces an accurate total displacement, but fails to represent the proper bending derivative behaviour around the interface cross point as well as near the right edge. 

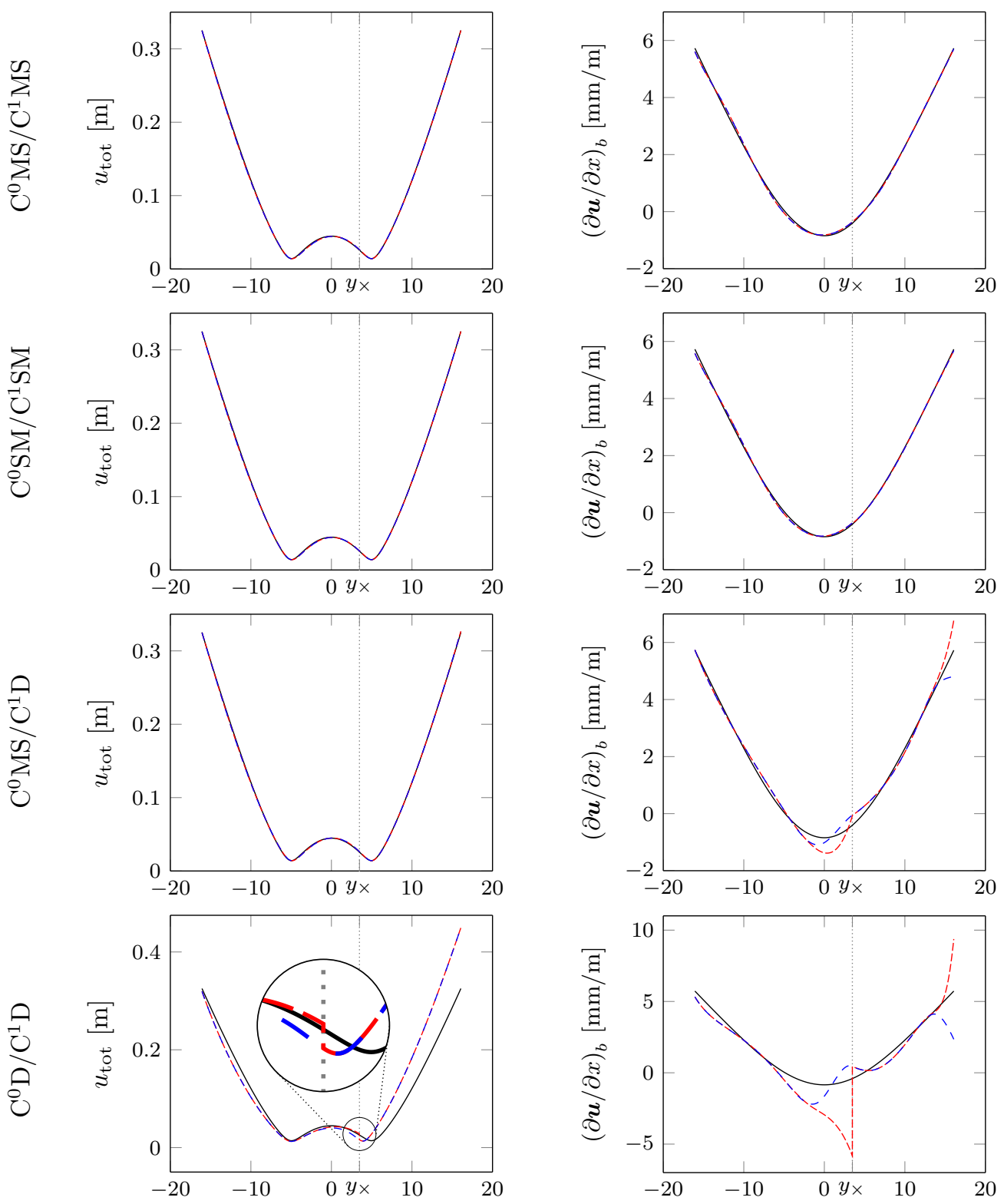

(a) Transverse displacement along the interface line.

(b) Bending component of the displacement's derivative normal to the interface.

Figure 11: Assessment of the solution accuracy on the green interface line of fig. 8c, comparing data from both sides of the interface $(--$ and ---$)$ to the reference solution $(-)$. The dotted vertical line marks the cross point $y_{\times}$of the green and the black interfaces in fig. $8 \mathrm{c}$.

- The $\mathrm{C}^{0} \mathrm{D} / \mathrm{C}^{1} \mathrm{D}$ total displacement shows a difference between both interface curves around the interface cross point, and also a jump for one of them. This means 
that there is a lack of continuity when crossing the interface cross point in either direction. Although there is $\mathrm{C}^{0}$-continuity away from the interface cross point, the corresponding total displacement is not accurate. This is linked to the fact that the $\mathrm{C}^{1}$-continuity of the bending component is completely distorted around the interface cross point and near the right edge.

This illustrates the importance of the use of a master-slave formulation in the case of non-conforming meshes, an observation which has also been made in [32] for the $\mathrm{C}^{0}$ coupling part. Although both the master-slave variants have performed similarly up till now, a proper choice of master and slave patch becomes more important as the mesh non-conformity increases.

\subsubsection{On the importance of choosing the coarser patch as the master}

The importance of using a proper master-slave coupling, with the coarser patch as the master, can be demonstrated by studying meshes with stronger non-conformities. Consider therefore the (cubic) mesh from fig. 12. For a given solution field $u_{\text {tot }}$, the errors

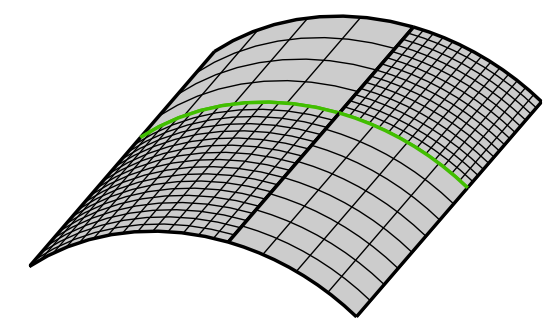

Figure 12: Strongly non-conforming four-patch mesh (cf. fig. 8c). The thick lines represent the patch boundaries; the green (curved) interfaces are studied in more detail.

in $\mathrm{C}^{0}$ - and $\mathrm{C}^{1}$-continuity across the green interface line of this mesh can be calculated according to the following (absolute) error criteria:

$$
\begin{aligned}
& \Delta \mathrm{C}_{\mathrm{int}}^{0}(y)=\left|u_{\mathrm{tot}}(y)\right|_{\mathrm{int}+}-\left.u_{\mathrm{tot}}(y)\right|_{\mathrm{int}-} \mid \\
& \Delta \mathrm{C}_{\mathrm{int}}^{1}(y)=\left|\left(\frac{\partial \boldsymbol{u}}{\partial x}(y)\right)_{b}\right|_{\mathrm{int}+}-\left.\left(\frac{\partial \boldsymbol{u}}{\partial x}(y)\right)_{b}\right|_{\text {int }-} \mid
\end{aligned}
$$

where $\left.\bullet\right|_{\text {int+ }}$ and $\left.\bullet\right|_{\text {int- }}$ refer to the multipatch model quantities evaluated using the variables belonging to either side of the green interfaces. It is important to note that these error criteria are not at all measures of the solution accuracy, but simply of the extent to which the continuity requirements are satisfied.

Figure 13 plots these errors for the two master-slave variants. These curves show that the proposed $\mathrm{C}^{0} \mathrm{MS} / \mathrm{C}^{1} \mathrm{MS}$ method fulfils the continuity requirements better than the alternative $\mathrm{C}^{0} \mathrm{SM} / \mathrm{C}^{1} \mathrm{SM}$ formulation, with a difference of several orders of magnitude. Particularly interesting to note for the $\mathrm{C}^{0} \mathrm{MS} / \mathrm{C}^{1} \mathrm{MS}$ model are the $\mathrm{C}^{0}$ error values near machine precision at the interface cross point $y_{\times}$as well as at the other interface edges. The locally interpolatory character of the NURBS basis functions makes this possible, together with the method's implicit one-to-one matching of the control variables at patch intersection points. This is not the case for the $\mathrm{C}^{0} \mathrm{SM} / \mathrm{C}^{1} \mathrm{SM}$ method, which has to use 


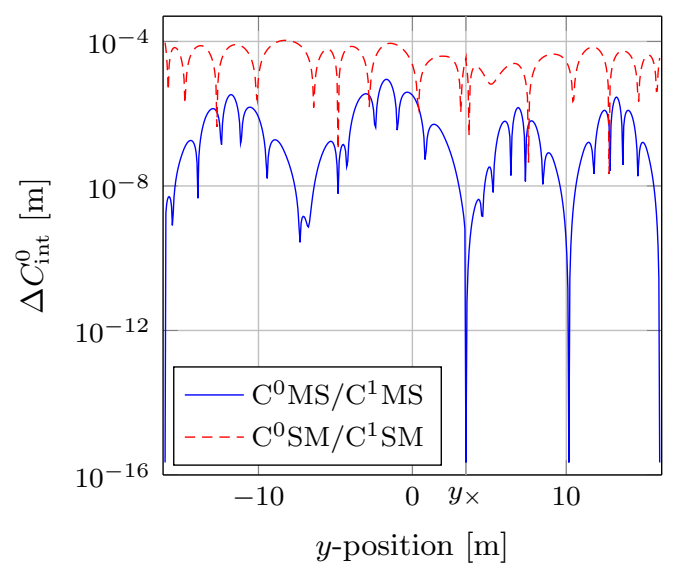

(a) $\mathrm{C}^{0}$-continuity error.

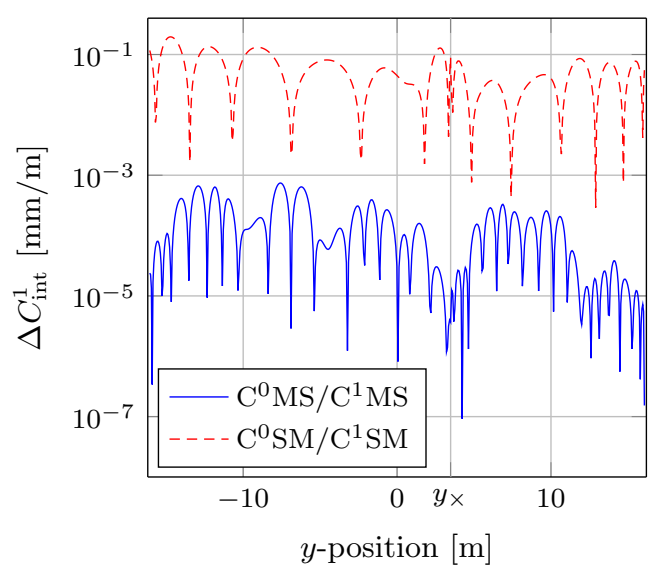

(b) $\mathrm{C}^{1}$-continuity error.

Figure 13: Absolute errors in (a) $\mathrm{C}^{0}$ - and (b) $\mathrm{C}^{1}$-continuity along the green interface line $(x=-5 \mathrm{~m})$ in fig. 12 for the two master-slave variants, according to eq. (31).

a smaller number of slave DOFs to match the behaviour of a larger number of master DOFs and thus has to relax on the one-to-one matching of the intersection DOFs.

Although satisfying the continuity requirements is necessary for obtaining an accurate solution field, the authors have shown in [32] that a lower error in continuity does not necessarily lead to an accurate solution. Figure 14 therefore plots the convergence of the relative error of the benchmark displacement for a series of strongly non-conforming meshes like the one in fig. 12. The error curves are generated using cubic NURBS basis functions. While the $\mathrm{C}^{0} \mathrm{SM} / \mathrm{C}^{1} \mathrm{SM}$ method's accuracy seems to stagnate, the proper choice of master and slave in the proposed method does allow for a converging solution even slightly superconvergent. This confirms the findings from [32] that using the coarser patch as the master yields more accurate and robust results.

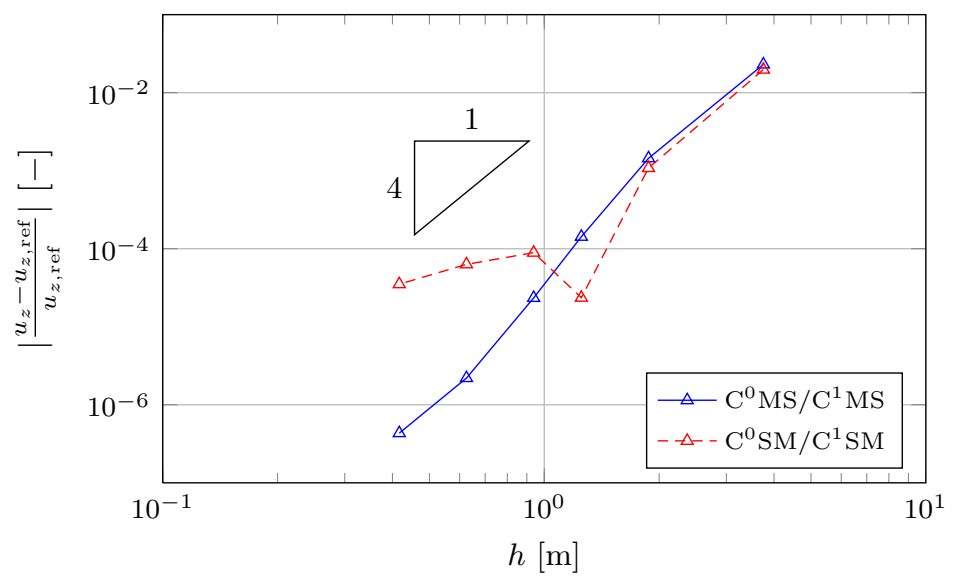

Figure 14: Convergence of the relative error of the benchmark displacement versus the average mesh size $h$ for the strongly non-conforming cubic meshes, obtained using the two different master-slave models. 


\subsection{Forced dynamic response of a curved structure containing kinks}

This example studies a curved structure composed of several shell parts connected in a non-smooth way, presenting a general patch coupling configuration. The structure is clamped at its bottom edges, as shown in fig. 15. It comprises six B-spline patches: the four patches forming the top part have curved interfaces and the clamped patches in addition meet their neighbours at a kink (indicated in green), i.e. a line without $\mathrm{G}^{1}$ continuity. A point force of $10 \mathrm{kN}$ vertically excites the structure at the centre of the outer edge. Because of this centred excitation together with the symmetric geometry and boundary conditions, the response should also be symmetric (with respect to the $x z$-plane).

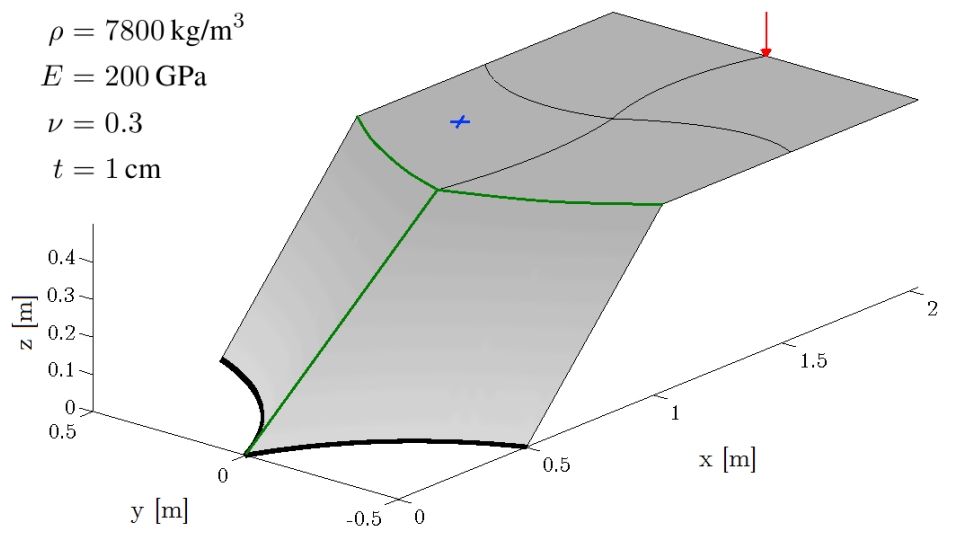

Figure 15: Kinked shell structure consisting of 6 patches (kinks highlighted in green). It is clamped at the bottom edge (thick black lines) and excited by a point force at the centre point of the outer boundary (indicated by a red arrow). The blue $\times$ is the response point for analysing the frequency behaviour.

The structure's dynamic response is studied in a frequency range between 0 and $500 \mathrm{~Hz}$, in particular in the response point indicated by the blue cross in fig. 15. Both a conforming and a non-conforming patch configuration (of almost exactly the same number of DOFs) are investigated, as shown in fig. 16. The models contain cubic B-spline patches, with 1014 elements in the conforming configuration and 1020 in the non-conforming one.

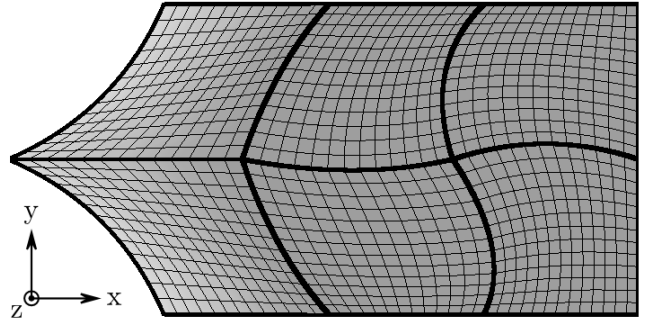

(a) Conforming configuration, containing 1014 elements with 4608 (uncoupled) DOFs.

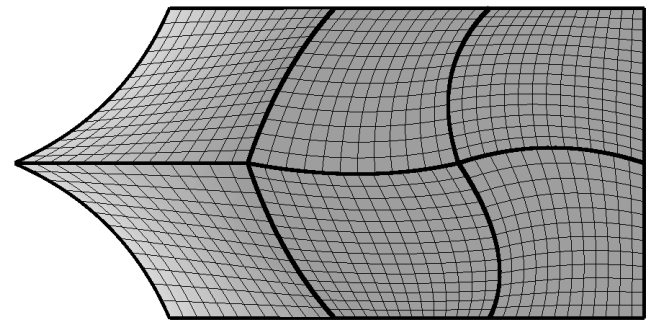

(b) Non-conforming configuration, containing 1020 elements with 4626 (uncoupled) DOFs.

Figure 16: The cubic B-spline meshes used for the isogeometric shell analysis of the kinked structure. The thick lines indicate the patch boundaries. 
This results in 4608 and 4626 uncoupled DOFs, respectively. After eliminating the dependent DOFs using the coupling equations, the respective models are left with 4175 and 4159 independent variables to be solved for. These isogeometric models are compared to an FE reference solution, computed using 20801 SHELL63 elements in ANSYS 14.5 [51]. With 6 DOFs per node for such shell elements, this leads to more than 126000 reference DOFs. Using a 10 elements per wavelength rule of thumb, this reference model is expected to be valid within engineering accuracy up to $2500 \mathrm{~Hz}$. Although this rule of thumb does not take into account pollution errors, these have a negligible influence in the studied frequency range.

Figure 17 plots the Frequency Response Function (FRF) for the vertical displacement amplitude in the response point (cf. fig. 15). Both isogeometric curves show good agreement with the reference solution over the entire frequency range. Only at the higher frequencies a very slight shift shows up in the response, slowly decreasing the solution accuracy. This is the onset of the well-known pollution effect [52]. Interesting to note is that, on FRF level in this frequency range, there does not seem to be a significant difference in performance between the conforming and the non-conforming mesh. Figure 18 confirms this by plotting the relative error for both isogeometric models. The two curves indeed show nearly exactly the same performance. As expected, the error increases with increasing frequency. There are some spikes in the curves, corresponding to the resonance and anti-resonance frequencies. This is due to very slight mismatches in peak frequency and for the anti-resonances additionally due to the very low reference values.

To assess the global accuracy of the IGA solutions, fig. 19 compares two plots of the total displacement amplitude (at 200 and $500 \mathrm{~Hz}$ ) obtained using the non-conforming model (cf. fig. 16b) and compares them to the FEM reference solution. Although the colour bars are slightly different between MATLAB and ANSYS, the correct deformation fields can be clearly recognised in figs. 19b and 19d. Note that even though the IGA mesh is non-symmetric (fig. 16b), the solutions are symmetric with respect to the $x z$-plane (as they should be). This illustrates that the asymmetric presence of the interfaces does not disturb the solution symmetry.

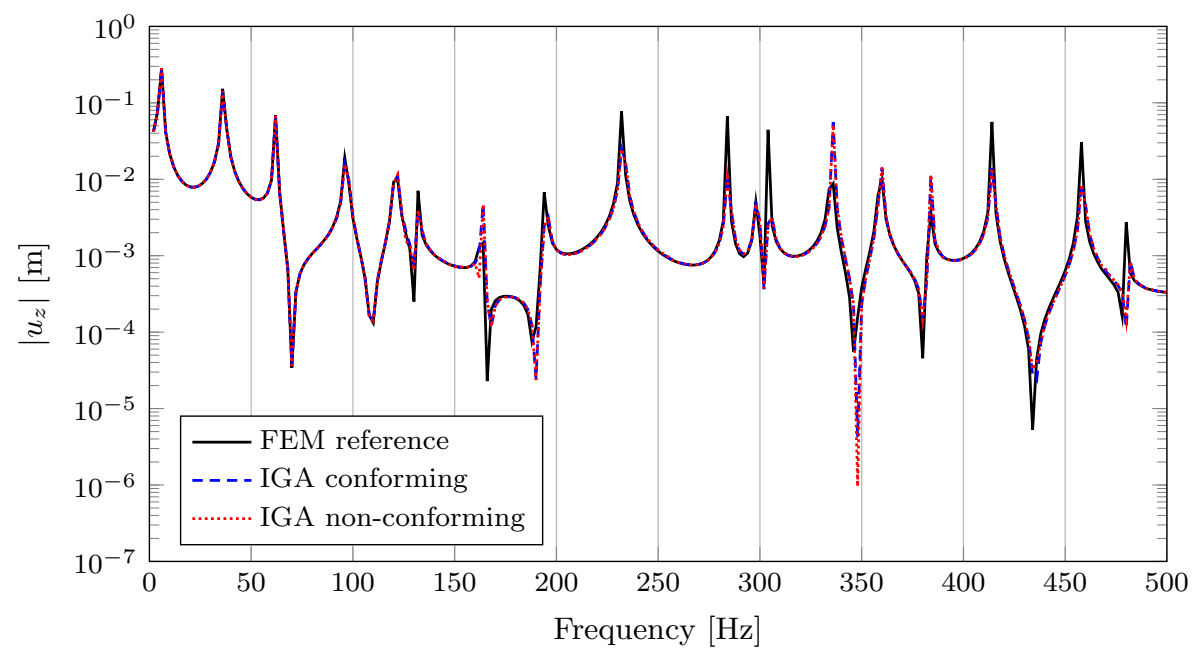

Figure 17: Amplitude of the vertical displacement in the response point in a frequency range between 0 and $500 \mathrm{~Hz}$ (with a frequency resolution of $2 \mathrm{~Hz}$ ), for the conforming and the nonconforming IGA model. 


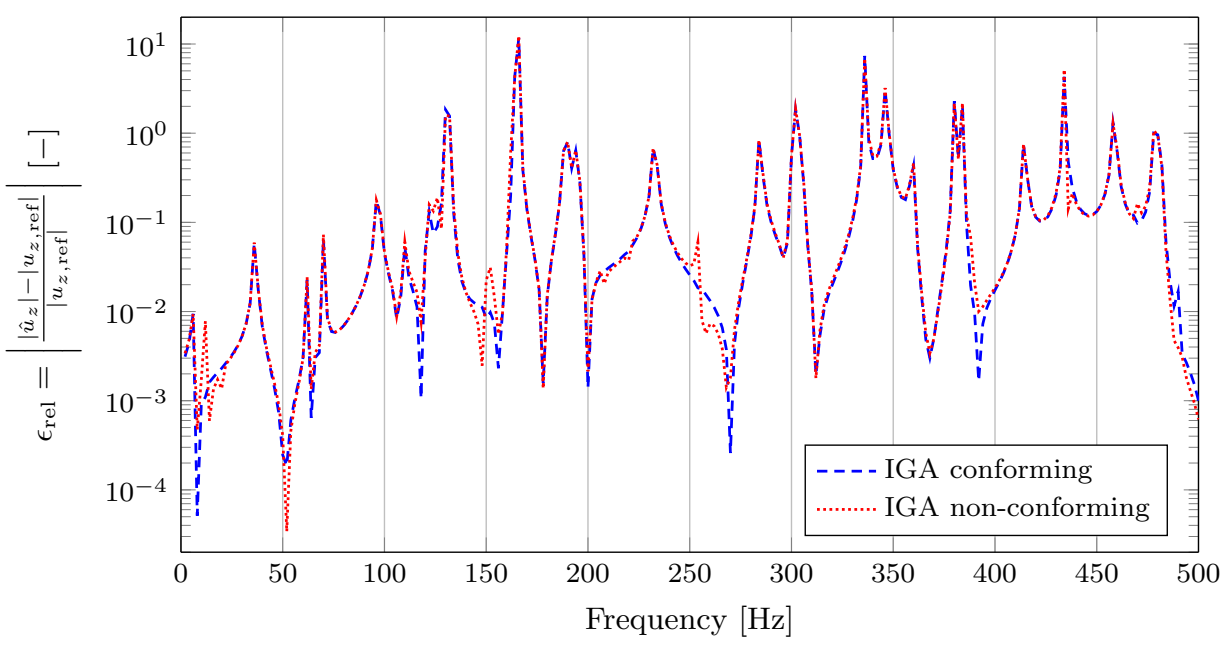

Figure 18: Relative error of the isogeometric FRF curves from fig. 17 with respect to the FEM reference solution.

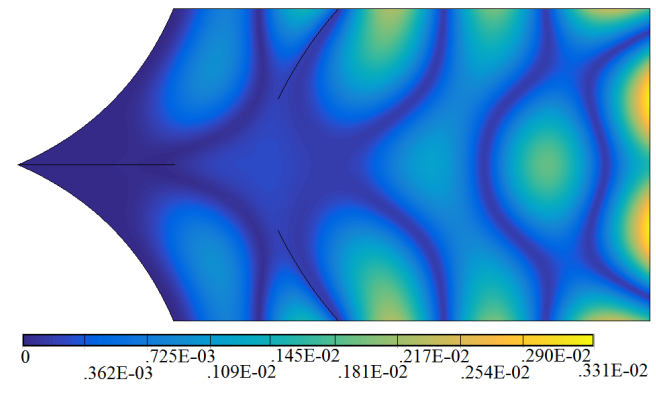

(a) FEM reference solution at $250 \mathrm{~Hz}$.

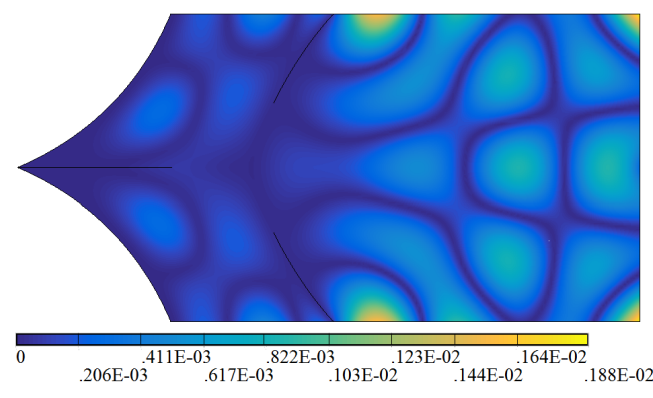

(c) FEM reference solution at $500 \mathrm{~Hz}$.

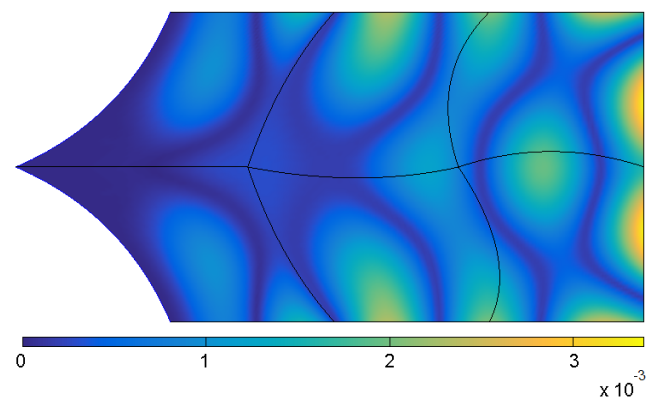

(b) IGA solution at $250 \mathrm{~Hz}$.

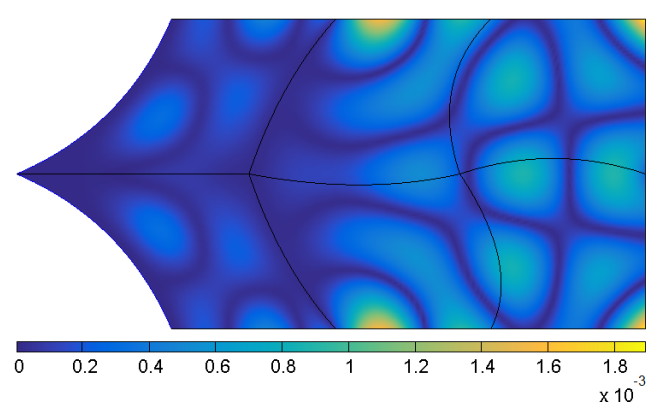

(d) IGA solution at $500 \mathrm{~Hz}$.

Figure 19: Total displacement amplitude [m] at 250 and $500 \mathrm{~Hz}$ for the non-conforming IGA model compared to the FEM reference solution.

\subsection{Forced dynamic response of a honeycomb sandwich panel}

As an example of a complex assembly of individual shell patches, this section studies a honeycomb sandwich panel. Such panels have a high stiffness-to-mass ratio, which 
makes them attractive for lightweight engineering structures. Their performance strongly depends on the cellular geometry of the core, in particular when attempting to achieve additional functionalities besides high stiffness or strength. Recent work has focused on obtaining acoustic stopband behaviour by adding local resonant structures inside the hollow core cells [53]. The interaction between these resonant inclusions and the hosting structure is crucial for the resulting acoustic insulation properties. However, honeycomb panels are typically modelled using a homogenised material with equivalent elastic properties derived from the geometric unit cell parameters [54]. Such a modelling concept does not allow for a smooth integration of geometric or material modifications at the level of the unit cell or smaller. The embedded parametrised geometric descriptions of IGA, on the other hand, allow for an efficient evaluation of design modifications even at a small scale. This property is particularly attractive when conducting optimisation studies of a full-size honeycomb sandwich panel.

Figure 20 shows the honeycomb sandwich panel under study here. It consists of two face sheets that are separated by a honeycomb core, comprising two full-size unit cells and six partial cells. All of the shell parts have a thickness $t=2 \mathrm{~mm}$ and material properties $E=200 \mathrm{GPa}, \nu=0.3$ and $\rho=7800 \mathrm{~kg} / \mathrm{m}^{3}$. For the numerical study, the panel is clamped in all of its free edges and excited by a point force. This force is oriented out-of-plane to the bottom face sheet and applied at the red cross-sign in fig. 20; the force magnitude is $1 \mathrm{kN}$. The dynamic response to this point force is studied in a frequency range up to $750 \mathrm{~Hz}$. In particular, the frequency response of the out-of-plane displacement is studied in the response point indicated by the blue plus-sign on the top face sheet in fig. 20 .

Figure 21 shows the B-spline mesh used for the isogeometric modelling of the panel. From a connectivity point-of-view, this is a highly complex model. It contains 39 patches (not all meeting under the same angle) interconnected by 112 single interfaces. Some of these interfaces coincide, resulting in 48 unique interfaces - most of which connect three patches. Some interface cross points even form the meeting point of seven different patches. The patches are discretised using quintic B-spline basis functions, as an illustration of the coupling technique using higher-order functions (and this higher polynomial order also allows a faster convergence). This leads to 11700 DOFs for the 975 elements of the entire structure; the patch coupling reduces this to 8814 independent DOFs.

The model from fig. 21 is verified against an FE reference model computed using

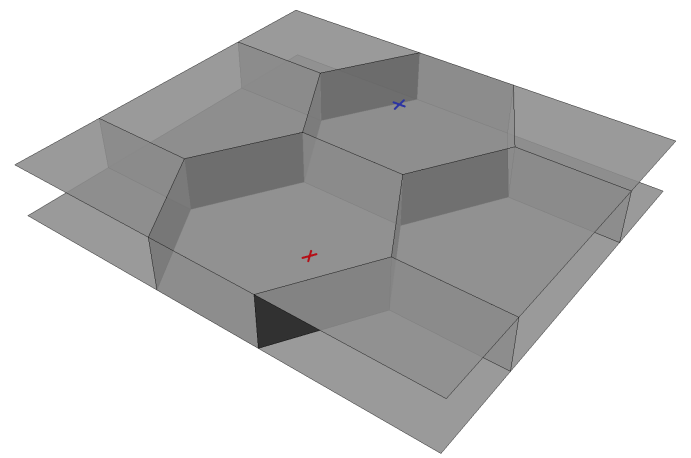

Figure 20: The honeycomb sandwich panel under study. Its outer box dimensions are $0.8 \mathrm{~m} \times$ $0.7 \mathrm{~m} \times 0.1 \mathrm{~m}$, with the sides of the hexagonal unit cells measuring $0.2 \mathrm{~m}$. All shells are $2 \mathrm{~mm}$ thick. The red $\times$ (on the bottom face sheet) indicates the force application point, the blue + (on the top face sheet) the FRF response point located at $(0.384 ; 0.450 ; 0.100) \mathrm{m}$. 


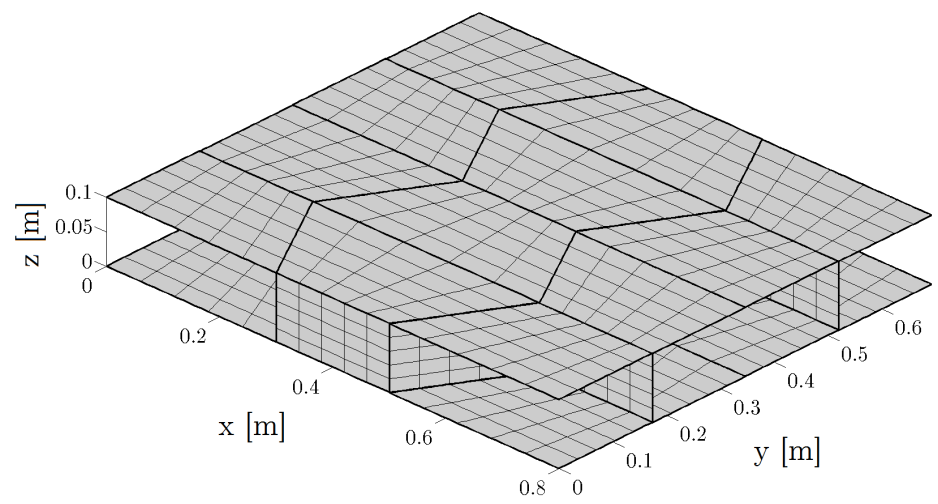

Figure 21: Isogeometric discretisation of the honeycomb panel, consisting of 39 B-spline patches connected by 48 (unique) interfaces between them. This results in 975 elements and (using quintic B-splines) 11700 uncoupled DOFs, yielding 8814 independent DOFs after the patch coupling.

ANSYS 14.5. It consists of 17503 SHELL63 elements with 6 DOFs per node, leading to 104976 reference DOFs. Using a 10 elements per wavelength rule of thumb, this reference model is expected to be valid within engineering accuracy up to $2000 \mathrm{~Hz}$. Although pollution errors are introduced already below this frequency, the FEM model is still more than adequate as a reference model for a qualitative verification of the proposed coupling method. Figure 22 plots the out-of-plane displacement amplitude in the response point (cf. fig. 20) for both the reference model and the isogeometric model from fig. 21. The curves show a good match between the isogeometric model and the reference. For the higher frequencies (above about $500 \mathrm{~Hz}$ ) the isogeometric curve starts to show a frequency shift introduced by the pollution effect, but the correct frequency behaviour is nonetheless still clearly present. Refining the B-spline discretisation can increase the accuracy further, as fig. 23 shows for a frequency range from 100 to $500 \mathrm{~Hz}$.

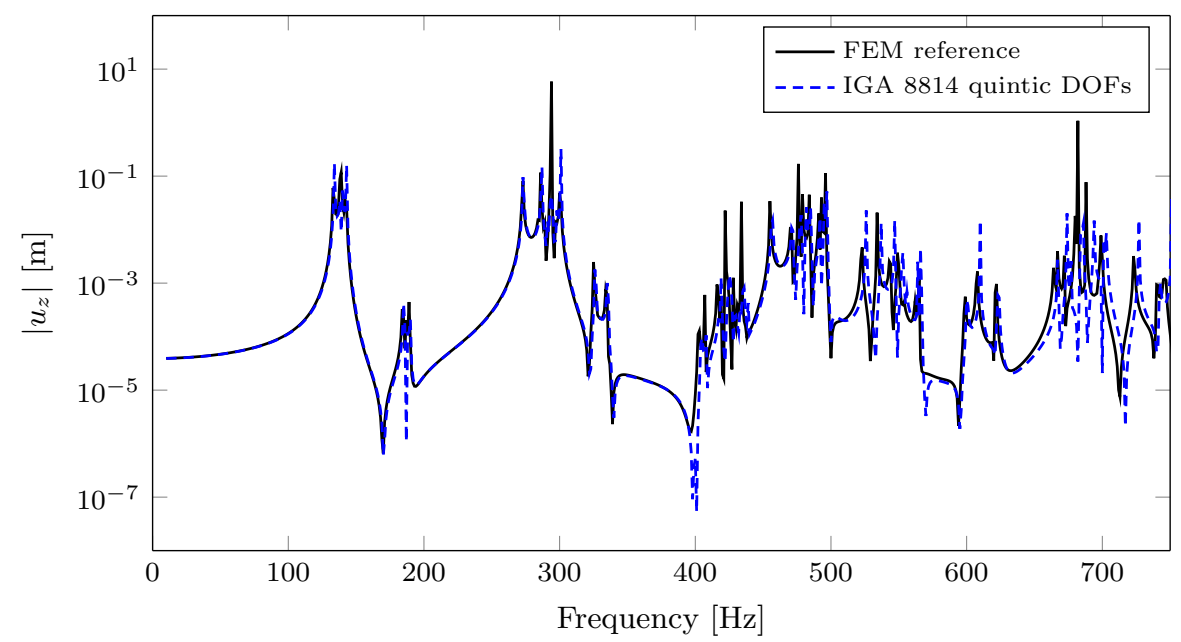

Figure 22: Amplitude of the vertical displacement in the response point in a frequency range from 10 to $750 \mathrm{~Hz}$ (with a frequency resolution of $1 \mathrm{~Hz}$ ). 


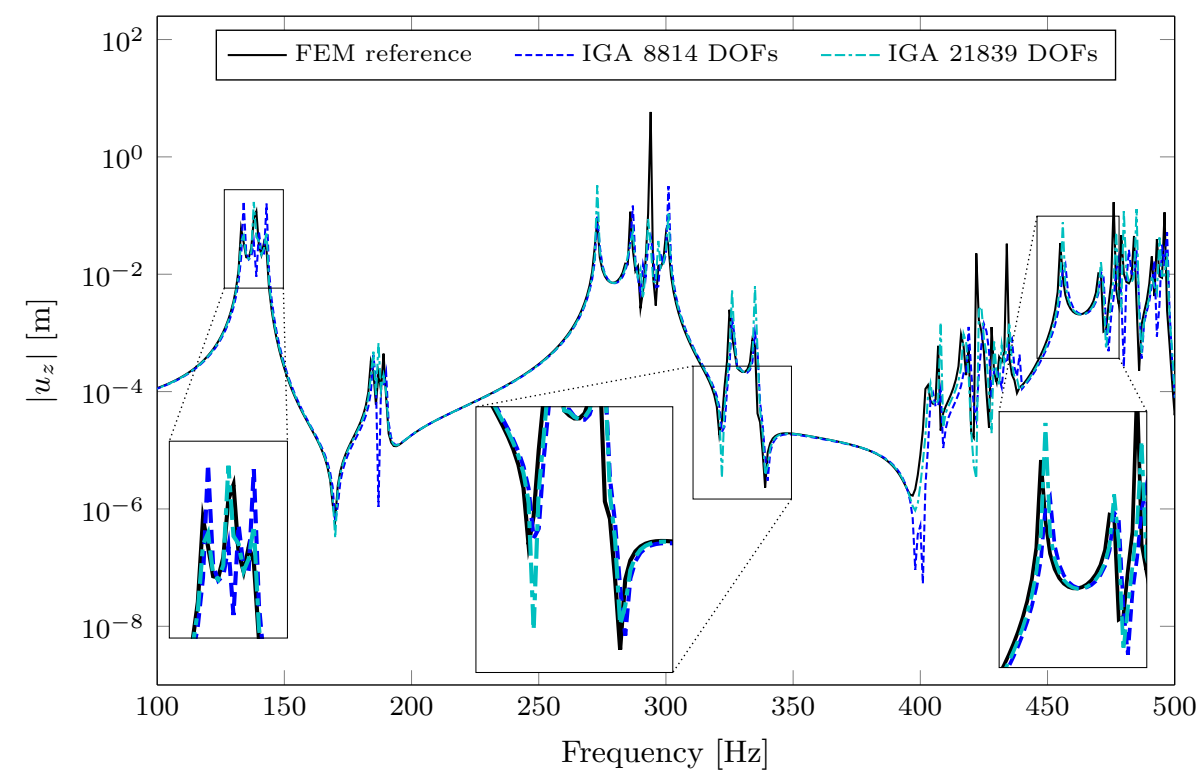

Figure 23: The FRF from fig. 22 in a frequency range from 100 to $500 \mathrm{~Hz}$, including an additional, more detailed IGA model of 21839 DOFs (still of order $p=5$ ).

\section{Conclusion}

This work presents a coupling method for imposing $\mathrm{C}^{1}$-continuity across NURBS patch boundaries in isogeometric Kirchhoff-Love shell analysis. Both the $\mathrm{C}^{0}$ - and the $\mathrm{C}^{1}$-parts of the proposed technique use a master-slave formulation for coupling non-conforming patches, but they differ in their approach. The $\mathrm{C}^{0}$-coupling imposes constraints in a weak sense, globally along the entire interface, whereas the $\mathrm{C}^{1}$-coupling explicitly matches first-order derivatives of the discretisation in certain well-chosen collocation points. More specifically, the $\mathrm{C}^{1}$-part of the coupling imposes the out-of-plane component of the gradient of the displacement field in the (in-plane) direction normal to the interface to be equal across the interface in a collocation-sense. Based on these $\mathrm{C}^{0}$ - and $\mathrm{C}^{1}$-constraints and on the choice for master and slave patches (where the coarser patch is chosen as the master), a corresponding set of dependent variables can be eliminated from the system of equations. This results in a reduced system, and can be done without any user interaction. No stabilisation or penalty parameters are involved. The coupling method is assessed using several numerical case studies of static as well as dynamic shell analysis, with both eigenvalue analyses and boundary-value problems. The geometries under study include curved patches as well as kink connections without $\mathrm{G}^{1}$-continuity. The proposed technique is found to yield accurate and converging results. Also non-conforming patch configurations, where even a $\mathrm{C}^{0}$-coupling is non-trivial, are investigated. For these configurations, the accuracy (as compared to conforming counterparts) decreases more for higher-order models than for lower-order ones.

\section{Acknowledgements}

The research of Laurens Coox is funded by a PhD grant from the Flanders Innovation \& Entrepreneurship Agency (VLAIO); that of Elke Deckers by a postdoctoral grant from 
the Research Foundation - Flanders (FWO). The European Commission is gratefully acknowledged for its support of the ANTARES research project (GA606817). The presented work is also partially supported by the Research Fund KU Leuven and by Flanders Make, the strategic research centre for the manufacturing industry.

\section{References}

[1] T. Hughes, J. Cottrell, Y. Bazilevs, Isogeometric analysis: CAD, finite elemens, NURBS, exact geometry and mesh refinement, Computer Methods in Applied Mechanics and Engineering 194 (2005) 4135-4195.

[2] J. Cottrell, T. Hughes, Y. Bazilevs, Isogeometric Analysis: Toward Integration of CAD and FEA, John Wiley \& Sons, 2009.

[3] V. P. Nguyen, C. Anitescu, S. P. Bordas, T. Rabczuk, Isogeometric analysis: an overview and computer implementation aspects, Mathematics and Computers in Simulation 117 (2015) 89-116.

[4] M. Bischoff, W. Wall, K.-U. Bletzinger, E. Ramm, Models and Finite Elements for Thin-Walled Structures, in: E. Stein, R. de Borst, T. Hughes (Eds.), Encyclopedia of Computational Mechanics, Vol. 2, John Wiley \& Sons, 2004, Ch. 3.

[5] H. Stolarski, T. Belytschko, S.-H. Lee, A review of shell finite elements and corotational theories, Computational Mechanics Advances 2 (1995) 125-212.

[6] E. Oñate, F. G. Flores, Advances in the formulation of the rotation-free basic shell triangle, Computational Methods for Shells 194 (2005) 2406-2443.

[7] N. Viebahn, P. M. Pimenta, J. Schröder, A simple triangular finite element for nonlinear thin shells: statics, dynamics and anisotropy, Computational Mechanics 59 (2017) 281-297.

[8] R. S. Falk, T. Tu, Locking-free finite elements for the Reissner-Mindlin plate, Mathematics of Computation 69 (1999) 911-928.

[9] G. M. Stanley, Continuum-based shell elements, Ph.D. thesis, Division of Applied Mechanics, Stanford University (1985).

[10] T. X. Duong, F. Roohbakhshan, R. A. Sauer, A new rotation-free isogeometric thin shell formulation and a corresponding continuity constraint for patch boundaries, Computer Methods in Applied Mechanics and Engineering 316 (2017) 43-83.

[11] J. Cottrell, T. Hughes, A. Reali, Studies of refinement and continuity in isogeometric structural analysis, Computer Methods in Applied Mechanics and Engineering 196 (2007) 4160-4183.

[12] S. K. Kleiss, C. Pechstein, B. Jüttler, S. Tomar, IETI - Isogeometric Tearing and Interconnecting, Computer Methods in Applied Mechanics and Engineering 247-248 (2012) 201-215.

[13] E. Brivadis, A. Buffa, B. Wohlmuth, L. Wunderlich, Isogeometric mortar methods, Computer Methods in Applied Mechanics and Engineering 284 (2015) 292-319. 
[14] W. Dornisch, G. Vitucci, S. Klinkel, The weak substitution method - an application of the mortar method for patch coupling in NURBS-based isogeometric analysis, International Journal for Numerical Methods in Engineering 103 (2015) 205-234.

[15] W. Dornisch, J. Stöckler, R. Müller, Dual and approximate dual basis functions for B-splines and NURBS - Comparison and application for an efficient coupling of patches with the isogeometric mortar method, Computer Methods in Applied Mechanics and Engineering 316 (2017) 449-496.

[16] V. Nguyen, P. Kerfriden, M. Brino, S. Bordas, E. Bonisoli, Nitsche's method for two and three dimensional NURBS patch coupling, Computational Mechanics 53 (2014) $1163-1182$.

[17] M. Ruess, D. Schillinger, A. Özcan, E. Rank, Weak coupling for isogeometric analysis of non-matching and trimmed multi-patch geometries, Computer Methods in Applied Mechanics and Engineering 269 (2014) 46-71.

[18] A. Apostolatos, R. Schmidt, R. Wüchner, K.-U. Bletzinger, A Nitsche-type formulation and comparison of the most common domain decomposition methods in isogeometric analysis, International Journal for Numerical Methods in Engineering 97 (2014) 473-504.

[19] C. Hesch, P. Betsch, Isogeometric analysis and domain decomposition methods, Computer Methods in Applied Mechanics and Engineering 213-216 (2012) 104-112.

[20] W. Dornisch, S. Klinkel, Treatment of Reissner-Mindlin shells with kinks without the need for drilling rotation stabilization in an isogeometric framework, Computer Methods in Applied Mechanics and Engineering 276 (2014) 35-66.

[21] P. M. Sobota, W. Dornisch, R. Müller, S. Klinkel, Implicit dynamic analysis using an isogeometric ReissnerMindlin shell formulation, International Journal for Numerical Methods in Engineering 110 (2017) 803-825.

[22] W. Dornisch, R. Müller, S. Klinkel, An efficient and robust rotational formulation for isogeometric Reissner-Mindlin shell elements, Computer Methods in Applied Mechanics and Engineering 303 (2016) 1-34.

[23] J. Kiendl, K.-U. Bletzinger, J. Linhard, R. Wüchner, Isogeometric shell analysis with Kirchhoff-Love elements, Computer Methods in Applied Mechanics and Engineering 198 (2009) 3902-3914.

[24] F. Maurin, L. Coox, F. Greco, E. Deckers, C. Claeys, W. Desmet, Bloch-Floquet theorem for isogeometric analysis of periodic problems governed by high-order partial differential equations, Computer Methods in Applied Mechanics and Engineering 311 (2016) 743-763.

[25] J. Kiendl, Y. Bazilevs, M.-C. Hsu, R. Wüchner, K.-U. Bletzinger, The bending strip method for isogeometric analysis of Kirchhoff-Love shell structures comprised of multiple patches, Computer Methods in Applied Mechanics and Engineering 199 (2010) 2403-2416.

[26] R. Schmidt, R. Wüchner, K.-U. Bletzinger, Isogeometric analysis of trimmed NURBS geometries, Computer Methods in Applied Mechanics and Engineering 241-244 (2012) 93-111. 
[27] A. Goyal, B. Simeon, On penalty-free formulations for multipatch isogeometric Kirchhoff-Love shells, Mathematics and Computers in Simulation 136 (2017) 78-103.

[28] S. Shojaee, E. Izadpanah, N. Valizadeh, J. Kiendl, Free vibration analysis of thin plates by using a NURBS-based isogeometric approach, Finite Elements in Analysis and Design 61 (2012) 23-34.

[29] A. Apostolatos, M. Breitenberger, R. Wüchner, K.-U. Bletzinger, Domain Decomposition Methods and Kirchhoff-Love Shell Multipatch Coupling in Isogeometric Analysis, Isogeometric Analysis and Applications 2014. Lecture Notes in Computational Science and Engineering 107 (2015) 73-101.

[30] Y. Guo, M. Ruess, Nitsche's method for a coupling of isogeometric thin shells and blended shell structures, Computer Methods in Applied Mechanics and Engineering 284 (2015) 881-905.

[31] M. Breitenberger, A. Apostolatos, B. Philipp, R. Wüchner, K.-U. Bletzinger, Analysis in computer aided design: Nonlinear isogeometric B-Rep analysis of shell structures, Computer Methods in Applied Mechanics and Engineering 284 (2015) 401-457.

[32] L. Coox, F. Greco, O. Atak, D. Vandepitte, W. Desmet, A robust patch coupling method for NURBS-based isogeometric analysis of non-conforming multipatch surfaces, Computer Methods in Applied Mechanics and Engineering 316 (2017) 235-260.

[33] L. Coox, O. Atak, D. Vandepitte, W. Desmet, An isogeometric indirect boundary element method for solving acoustic problems in open-boundary domains, Computer Methods in Applied Mechanics and Engineering 316 (2017) 186-208.

[34] L. Piegl, W. Tiller, The NURBS Book, Springer-Verlag Berlin Heidelberg, 1997.

[35] M. Cox, The numerical evaluation of B-splines, DNAC 4, National Physics Laboratory (1971).

[36] C. de Boor, On calculation with B-splines, Journal of Approximation Theory 6 (1972) $50-62$.

[37] R. Craig, A. Kurdila, Fundamentals of Structural Dynamics, John Wiley \& Sons, Inc., 2006.

[38] J. Kiendl, M.-C. Hsu, M. C. Wu, A. Reali, Isogeometric Kirchhoff-Love shell formulations for general hyperelastic materials, Computer Methods in Applied Mechanics and Engineering 291 (2015) 280-303.

[39] C. de Boor, A practical guide to splines, Springer-Verlag, 1979.

[40] F. Auricchio, L. Beir ao da Veiga, T. Hughes, A. Reali, G. Sangalli, Isogeometric collocation methods, Mathematical Models and Methods in Applied Sciences 10 (2010) 2075-2107.

[41] D. Schillinger, J. A. Evans, A. Reali, M. A. Scott, T. Hughes, Isogeometric collocation: Cost comparison with Galerkin methods and extension to adaptive hierarchical NURBS discretizations, Computer Methods in Applied Mechanics and Engineering 267 (2013) 170-232. 
[42] S. Demko, On the existence of interpolation projectors onto spline spaces, Journal of Approximation Theory 43 (1985) 151-156.

[43] H. Gomez, L. De Lorenzis, The variational collocation method, Computer Methods in Applied Mechanics and Engineering 309 (2016) 152-181.

[44] T. F. Chan, P. C. Hansen, Some applications of the rank-revealing QR factorization, SIAM Journal on Scientific Computing 13 (1992) 727-741.

[45] J. Reddy, Theory and Analysis of Elastic Plates and Shells, CRC Press, 2007.

[46] T. Hughes, J. A. Evans, A. Reali, Finite element and NURBS approximations of eigenvalue, boundary-value and initial-value problems, Computer Methods in Applied Mechanics and Engineering 272 (2014) 290-320.

[47] J. Cottrell, A. Reali, Y. Bazilevs, T. Hughes, Isogeometric analysis of structural vibrations, Computer Methods in Applied Mechanics and Engineering 195 (2006) $5257-5296$.

[48] L. Coox, E. Deckers, D. Vandepitte, W. Desmet, A performance study of NURBSbased isogeometric analysis for interior two-dimensional time-harmonic acoustics, Computer Methods in Applied Mechanics and Engineering 305 (2016) 441-467.

[49] A. Tagliabue, L. Dedè, A. Quarteroni, Isogeometric Analysis and error estimates for high order partial differential equations in fluid dynamics, Computers \& Fluids 102 (2014) 277-303.

[50] T. Belytschko, H. Stolarski, W. Liu, N. Carpenter, J.-J. Ong, Stress projection for membrane and shear locking in shell finite elements, Computer Methods in Applied Mechanics and Engineering 51 (1985) 221-258.

[51] ANSYS, Inc., ANSYS Structural Mechanics (Jan. 2017).

URL http://www.ansys.com/products/structures

[52] P. Bouillard, F. Ihlenburg, Error estimation and adaptivity for the finite element method in acoustics: 2D and 3D applications, Computer Methods in Applied Mechanics and Engineering 176 (1999) 147-163.

[53] C. Claeys, E. Deckers, B. Pluymers, W. Desmet, A lightweight vibro-acoustic metamaterial demonstrator: Numerical and experimental investigation, Mechanical Systems and Signal Processing 70-71 (2016) 853-880.

[54] L. J. Gibson, M. F. Ashby, Cellular Solids: Structure and Properties, Cambridge University Press, 1999. 